\title{
Total Synthesis of (+)-Macquarimicin A
}

\author{
Ryosuke Munakata, Hironori Katakai, Tatsuo Ueki, Jun Kurosaka, Ken-ichi Takao, Kin-ichi Tadano* \\ Department of Applied Chemistry, Keio University, Hiyoshi, Kohoku-ku, Yokohama 223-8522, Japan
}

\section{Supporting Information}

\section{Experimental Section}

\section{General}

Specific rotations were measured in a $10 \mathrm{~mm}$ cell. ${ }^{1} \mathrm{H}$ NMR spectra were recorded at $270 \mathrm{MHz}$ or at 300 $\mathrm{MHz}$ with tetramethylsilane as an internal standard. ${ }^{13} \mathrm{C}$ NMR spectra were recorded at $68 \mathrm{MHz}$ or at $75 \mathrm{MHz}$. All spectra were recorded in $\mathrm{CDCl}_{3}$ as solvent. High-resolution mass spectra (HRMS) were measured by the EI method $(70 \mathrm{eV})$ unless otherwise noted. Thin-layer chromatography (TLC) was performed with a glass plate coated with Kieselgel $60 \mathrm{~F}_{254}$ (Merck). The crude reaction mixtures and extractive materials were purified by chromatography on silica gel Daisogel IR-60 (Daiso Co., Ltd.) or Wakogel C300 (Wako Pure Chemical Industries). Unless otherwise described, reactions were carried out at ambient temperature. Combined organic extracts were dried over anhydrous $\mathrm{Na}_{2} \mathrm{SO}_{4}$. Solvents were removed from reaction mixture or combined organic extracts by concentration under reduced pressure using an evaporator with a water bath at $35-45^{\circ} \mathrm{C}$.

(1Z,3S,4R,6E)-1-Bromo-8-(t-butyldiphenylsilyloxy)-3-[(4-methoxyphenyl)methoxy]-4-methyl-1,6-octadi ene (10)

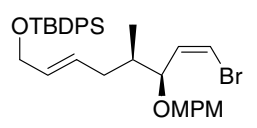

The following reaction was carried out under argon. To a stirred solution of $\mathrm{PPh}_{3}(764 \mathrm{mg}, 2.91 \mathrm{mmol})$ in degassed toluene $(160 \mathrm{~mL})$ was added $\mathrm{Pd}(\mathrm{OAc})_{2}(163 \mathrm{mg}, 0.724 \mathrm{mmol})$. The mixture was stirred for $40 \mathrm{~min}$ and then $9^{1}$ (12.2 g, $\left.18.1 \mathrm{mmol}\right)$ in degassed toluene $(80 \mathrm{~mL})$ and $\mathrm{Bu}_{3} \mathrm{SnH}(5.4 \mathrm{~mL}, 20.1 \mathrm{mmol})$ were added. The mixture was stirred for $30 \mathrm{~min}$ and then diluted with EtOAc $(250 \mathrm{~mL})$. This was washed with saturated brine. The combined aqueous layers were extracted with EtOAc. The combined organic layers were dried and concentrated in vacuo. The residue was purified by column chromatography on silica gel $\left(\mathrm{Et}_{3} \mathrm{~N} /\right.$ hexane, 1:100 then EtOAc/hexane, 1:70) to provide $9.19 \mathrm{~g}(85 \%)$ of $\mathbf{1 0}$ as a colorless oil; TLC, $\mathrm{R}_{f} 0.68$ (EtOAc/hexane, 1:3); $[\alpha]^{24}{ }_{\mathrm{D}}+3.00\left(c 1.72, \mathrm{CHCl}_{3}\right) ;{ }^{1} \mathrm{H}$ NMR $(270 \mathrm{MHz}) \delta 0.95(\mathrm{~d}, J=6.6 \mathrm{~Hz}, 3 \mathrm{H}), 1.05(\mathrm{~s}, 9 \mathrm{H}), 1.75(\mathrm{~m}, 1 \mathrm{H})$, $1.87(\mathrm{~m}, 1 \mathrm{H}), 2.24(\mathrm{~m}, 1 \mathrm{H}), 3.78(\mathrm{~s}, 3 \mathrm{H}), 4.14(\mathrm{~d}, J=4.4 \mathrm{~Hz}, 2 \mathrm{H}), 4.17(\mathrm{dd}, J=5.9,8.8 \mathrm{~Hz}, 1 \mathrm{H}), 4.28,4.50$

\footnotetext{
${ }^{1}$ Munakata, R.; Ueki, T.; Katakai, H.; Takao, K.; Tadano, K. Org. Lett. 2001, 3, 3029.
} 
$(2 \mathrm{~d}, J=11.5 \mathrm{~Hz}, 1 \mathrm{H} \times 2), 5.52(\mathrm{dt}, J=15.4,4.4 \mathrm{~Hz}, 1 \mathrm{H}), 5.63(\mathrm{dt}, J=15.4,6.4 \mathrm{~Hz}, 1 \mathrm{H}), 6.14(\mathrm{dd}, J=7.3,8.8$ $\mathrm{Hz}, 1 \mathrm{H}), 6.42(\mathrm{dd}, J=0.7,7.3 \mathrm{~Hz}, 1 \mathrm{H}), 6.86(\mathrm{~m}, 2 \mathrm{H}), 7.26(\mathrm{~m}, 2 \mathrm{H}), 7.36-7.40(\mathrm{~m}, 6 \mathrm{H}), 7.66-7.70(\mathrm{~m}, 4 \mathrm{H}) ;{ }^{13} \mathrm{C}$ NMR $(68 \mathrm{MHz}) \delta 14.94,19.21,26.81 \times 3,35.39,37.78,55.20,64.45,70.38,79.28,110.58,113.63 \times 2$, $127.60 \times 4,128.98,129.41 \times 2,129.53 \times 2,130.53,130.68,133.85 \times 2,135.11,135.52 \times 4,159.04$; IR (neat) 3080 , 2940, 1615, $1515 \mathrm{~cm}^{-1}$; HRMS calcd for $\mathrm{C}_{29} \mathrm{H}_{32} \mathrm{O}_{3} \mathrm{Si}^{81} \mathrm{Br}\left(\mathrm{M}^{+}-t-\mathrm{Bu}\right) \mathrm{m} / \mathrm{z} 537.1284$, found 537.1287.

$(1 Z, 3 S, 4 R, 6 E)-8$-( $t$-Butyldiphenylsilyloxy)-3-[(4-methoxyphenyl)methoxy]-4-methyl-1-tributylstannyl-1, 6-octadiene (7)

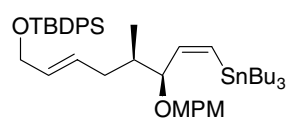

The following reaction was carried out under argon. To a cooled $\left(-78^{\circ} \mathrm{C}\right)$, stirred solution of $10(9.19 \mathrm{~g}, 15.5$ mmol) in $\mathrm{Et}_{2} \mathrm{O}(180 \mathrm{~mL})$ was added $t$ - $\mathrm{BuLi}(27 \mathrm{~mL}$ of $1.40 \mathrm{M}$ in pentane, $37 \mathrm{mmol})$. The mixture was stirred at $-78{ }^{\circ} \mathrm{C}$ for $1 \mathrm{~h}$ and then $\mathrm{Bu}_{3} \mathrm{SnCl}(5.1 \mathrm{~mL}, 18.6 \mathrm{mmol})$ was added. The mixture was stirred at $-78{ }^{\circ} \mathrm{C}$ for 2 $h$ and then quenched with $\mathrm{H}_{2} \mathrm{O}$. This was diluted with EtOAc $(250 \mathrm{~mL})$, and washed with $\mathrm{H}_{2} \mathrm{O}$. The organic layer was dried and concentrated in vacuo. The residue was purified by column chromatography on silica gel $\left(\mathrm{Et}_{3} \mathrm{~N} /\right.$ hexane, 1:100 then EtOAc/hexane, 1:100) to provide $9.96 \mathrm{~g}(80 \%)$ of 7 as a colorless oil; TLC, $\mathrm{R}_{f} 0.77$ (EtOAc/hexane, 1:5); $[\alpha]^{24}+5.23\left(c 1.99, \mathrm{CHCl}_{3}\right) ;{ }^{1} \mathrm{H}$ NMR $(300 \mathrm{MHz}) \delta 0.85-0.91(\mathrm{~m}, 15 \mathrm{H}), 0.96(\mathrm{~d}, J=$ $6.8 \mathrm{~Hz}, 3 \mathrm{H}), 1.05(\mathrm{~s}, 9 \mathrm{H}), 1.25-1.33(\mathrm{~m}, 6 \mathrm{H}), 1.42-1.50(\mathrm{~m}, 6 \mathrm{H}), 1.67(\mathrm{~m}, 1 \mathrm{H}), 1.86(\mathrm{~m}, 1 \mathrm{H}), 2.30(\mathrm{~m}, 1 \mathrm{H})$, $3.45(\mathrm{dd}, J=4.9,8.8 \mathrm{~Hz}, 1 \mathrm{H}), 3.79(\mathrm{~s}, 3 \mathrm{H}), 4.15(\mathrm{~d}, J=3.9 \mathrm{~Hz}, 2 \mathrm{H}), 4.22,4.51(2 \mathrm{~d}, J=11.4 \mathrm{~Hz}, 1 \mathrm{H} \times 2)$, $5.51-5.68(\mathrm{~m}, 2 \mathrm{H}), 6.15\left(\mathrm{~d}, J=13.1,{ }^{2} J_{\mathrm{Sn}-\mathrm{H}}=62.7 \mathrm{~Hz}, 1 \mathrm{H}\right), 6.42(\mathrm{dd}, J=8.8,13.1 \mathrm{~Hz}, 1 \mathrm{H}), 6.86(\mathrm{~m}, 2 \mathrm{H}), 7.24$ $(\mathrm{m}, 2 \mathrm{H}), 7.33-7.42(\mathrm{~m}, 6 \mathrm{H}), 7.66-7.70(\mathrm{~m}, 4 \mathrm{H}) ;{ }^{13} \mathrm{C} \mathrm{NMR}(75 \mathrm{MHz}) \delta 10.51 \times 3,13.68 \times 3,14.59,19.20$, $26.80 \times 3,27.34 \times 3,29.15 \times 3,36.32,38.95,55.24,64.61,69.73,86.15,113.65 \times 2,127.57 \times 4,128.78 \times 2$, $129.52 \times 2,129.95,130.21,131.25,132.81,133.85 \times 2,135.53 \times 4,148.49,158.87$; IR (neat) 3080, 2960, 1615, $1515 \mathrm{~cm}^{-1}$; HRMS calcd for $\mathrm{C}_{41} \mathrm{H}_{59} \mathrm{O}_{3} \mathrm{Si}^{118} \mathrm{Sn}\left(\mathrm{M}^{+}-\mathrm{Bu}\right) \mathrm{m} / \mathrm{z} 745.3252$, found 745.3241.

(2R)-1-Chloro-5-trimethylsilyl-4-pentyn-2-ol (12 $)^{2}$

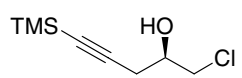

The following reaction was carried out under argon. To a cooled $\left(-78{ }^{\circ} \mathrm{C}\right)$, stirred solution of trimethylsilylacetylene $(5.0 \mathrm{~mL}, 35 \mathrm{mmol})$ in THF $(45 \mathrm{~mL})$ was added $n$-BuLi (13 mL of $2.66 \mathrm{M}$ in hexane, $35 \mathrm{mmol})$. The mixture was stirred at $-78{ }^{\circ} \mathrm{C}$ for $10 \mathrm{~min}$ and then $(R)$-epichlorohydrin $(\mathbf{1 1})(2.3 \mathrm{~mL}, 30$ mmol) was added. The mixture was stirred at $-78{ }^{\circ} \mathrm{C}$ for $10 \mathrm{~min}$ and then $\mathrm{BF}_{3} \cdot \mathrm{Et}_{2} \mathrm{O}(4.9 \mathrm{~mL}, 35 \mathrm{mmol})$ was added. The mixture was stirred while gradually warmed to $-30{ }^{\circ} \mathrm{C}$ over $2 \mathrm{~h}$ and at $-30{ }^{\circ} \mathrm{C}$ for $19 \mathrm{~h}$. The mixture was quenched with saturated aqueous $\mathrm{NH}_{4} \mathrm{Cl}$. This was diluted with saturated aqueous $\mathrm{NH}_{4} \mathrm{Cl}(200$ $\mathrm{mL}$ ), and extracted with EtOAc. The combined organic layers were dried and concentrated in vacuo to give crude 12, which was used in the next step without purification. In a small-scale experiment, pure $\mathbf{1 2}$ was

2 Takano, S.; Kamikubo, T.; Sugihara, T.; Suzuki, M.; Ogasawara, K. Tetrahedron: Asymmetry 1993, 4, 201. 
obtained by column chromatography on silica gel (EtOAc/hexane, 1:20) as a colorless oil; TLC, $\mathrm{R}_{f} 0.60$ (EtOAc/hexane, 1:2); $[\alpha]^{19}{ }_{\mathrm{D}}-10.7\left(c 1.69, \mathrm{CHCl}_{3}\right) ;{ }^{1} \mathrm{H}$ NMR $(270 \mathrm{MHz}) \delta 0.16(\mathrm{~s}, 9 \mathrm{H}), 2.54$ (dd, $J=6.6$, $16.9 \mathrm{~Hz}, 1 \mathrm{H}), 2.55(\mathrm{~m}, 1 \mathrm{H}), 2.62(\mathrm{dd}, J=5.9,16.9 \mathrm{~Hz}, 1 \mathrm{H}), 3.62(\mathrm{dd}, J=6.0,11.4 \mathrm{~Hz}, 1 \mathrm{H}), 3.72(\mathrm{dd}, J=4.4$, $11.4 \mathrm{~Hz}, 1 \mathrm{H}), 3.98(\mathrm{~m}, 1 \mathrm{H}) ;{ }^{13} \mathrm{C}$ NMR $(68 \mathrm{MHz}) \delta-0.03 \times 3,25.74,48.23,69.60,88.26,101.22$; IR (neat) $3400,2960,2180 \mathrm{~cm}^{-1}$.

(3R)-3-Hydroxy-6-trimethylsilyl-5-hexynenitrile and (3R)-3-Hydroxy-5-hexynenitrile

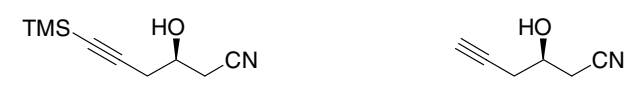

To a stirred solution of the crude $\mathbf{1 2}$ obtained above in DMSO- $\mathrm{H}_{2} \mathrm{O}(10: 1,44 \mathrm{~mL})$ were added $\mathrm{KCN}(2.88 \mathrm{~g}$, $44.3 \mathrm{mmol})$ and $\mathrm{NaI}(13.2 \mathrm{~g}, 88.5 \mathrm{mmol})$. The mixture was stirred at $80{ }^{\circ} \mathrm{C}$ for $5 \mathrm{~h}$ and then diluted with EtOAc $(250 \mathrm{~mL})$. This was washed with saturated brine. The combined aqueous layers were extracted with EtOAc. The combined organic layers were dried and concentrated in vacuo to give crude mixture of the nitriles, which were used in the next step without purification. In a small-scale experiment, pure products were obtained by column chromatography on silica gel (EtOAc/hexane, 1:15 to 1:3) as a colorless oil; (3R)-3-Hydroxy-6-trimethylsilyl-5-hexynenitrile: TLC, $\mathrm{R}_{f} 0.30$ (EtOAc/hexane, 1:3); ${ }^{1} \mathrm{H}$ NMR (300 MHz) $\delta$ $0.17(\mathrm{~s}, 9 \mathrm{H}), 2.39(\mathrm{~d}, J=5.8 \mathrm{~Hz}, 1 \mathrm{H}), 2.59(\mathrm{~d}, J=6.0 \mathrm{~Hz}, 2 \mathrm{H}), 2.66(\mathrm{~m}, 2 \mathrm{H}), 4.11(\mathrm{~m}, 1 \mathrm{H}) ;{ }^{13} \mathrm{C} \mathrm{NMR}(68$ MHz) $\delta-0.12 \times 3,24.73,28.02,66.09,89.56,100.18,117.11$; IR (neat) 3450, 2960, 2250, $2180 \mathrm{~cm}^{-1}$; (3R)-3-Hydroxy-5-hexynenitrile: TLC, $\mathrm{R}_{f} 0.16$ (EtOAc/hexane, 1:3); $[\alpha]^{23}{ }_{\mathrm{D}}-22.0\left(c\right.$ 2.06, $\left.\mathrm{CHCl}_{3}\right)$; ${ }^{1} \mathrm{H} \mathrm{NMR}$ $(270 \mathrm{MHz}) \delta 2.15(\mathrm{t}, J=2.6 \mathrm{~Hz}, 1 \mathrm{H}), 2.39(\mathrm{~d}, J=5.5 \mathrm{~Hz}, 1 \mathrm{H}), 2.57(\mathrm{dd}, J=2.6,5.9 \mathrm{~Hz}, 2 \mathrm{H}), 2.65(\mathrm{dd}, J=$ 6.4, $16.8 \mathrm{~Hz}, 1 \mathrm{H}), 2.72(\mathrm{dd}, J=5.5,16.8 \mathrm{~Hz}, 1 \mathrm{H}), 4.15(\mathrm{~m}, 1 \mathrm{H}) ;{ }^{13} \mathrm{C}$ NMR $(68 \mathrm{MHz}) \delta 24.62,26.38,65.88$, 72.19, 78.56, 117.32; IR (neat) 3450, 3300, 2920, 2250, $2120 \mathrm{~cm}^{-1}$; HRMS calcd for $\mathrm{C}_{6} \mathrm{H}_{8} \mathrm{NO}\left(\mathrm{M}^{+}+\mathrm{H}\right) \mathrm{m} / \mathrm{z}$ 110.0606 , found 110.0628 .

\section{(3R)-3-Hydroxy-5-hexynenitrile}

$$
\overbrace{}^{\mathrm{HO}} \mathrm{CN}
$$

To a cooled $\left(0{ }^{\circ} \mathrm{C}\right)$, stirred solution of the crude mixture obtained above in $\mathrm{MeOH}(26 \mathrm{~mL})$ was added $\mathrm{K}_{2} \mathrm{CO}_{3}$ (395 mg, $2.86 \mathrm{mmol}$ ). The mixture was stirred for $4.5 \mathrm{~h}$ and then Amberlite IR $120\left[\mathrm{H}^{+}\right]$was added at $0{ }^{\circ} \mathrm{C}$ until the $\mathrm{pH}$ of the mixture showed 6 . The resin was filtered off and the filtrate was concentrated in vacuo to give the crude nitrile, which was used in the next step without purification.

\section{(3R)-3-(t-Butyldimethylsilyloxy)-5-hexynenitrile}

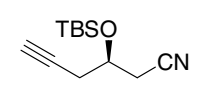

To a cooled $\left(0{ }^{\circ} \mathrm{C}\right)$, stirred solution of the crude (3R)-3-hydroxy-5-hexynenitrile obtained above in DMF (25 $\mathrm{mL})$ were added imidazole $(8.02 \mathrm{~g}, 118 \mathrm{mmol})$ and TBSCl $(8.92 \mathrm{~g}, 59.2 \mathrm{mmol})$. The mixture was stirred for $13.5 \mathrm{~h}$ and then diluted with $\mathrm{H}_{2} \mathrm{O}(300 \mathrm{~mL})$. This was extracted with $\mathrm{CH}_{2} \mathrm{Cl}_{2}$. The combined organic layers 
were dried and concentrated in vacuo. The residue was purified by column chromatography on silica gel (EtOAc/hexane, 1:40) to provide $5.62 \mathrm{~g}\left(84 \%, 4\right.$ steps from 11) of the TBS ether as a colorless oil; TLC, $\mathrm{R}_{f}$ 0.67 (EtOAc/hexane, 1:2); $[\alpha]^{23}{ }_{\mathrm{D}}-16.7\left(c 2.06, \mathrm{CHCl}_{3}\right) ;{ }^{1} \mathrm{H}$ NMR $(270 \mathrm{MHz}) \delta 0.12,0.15(2 \mathrm{~s}, 3 \mathrm{H} \times 2), 0.91$ (s, 9H), $2.07(\mathrm{t}, J=2.6 \mathrm{~Hz}, 1 \mathrm{H}), 2.43(\mathrm{ddd}, J=2.6,7.3,16.9 \mathrm{~Hz}, 1 \mathrm{H}), 2.51(\mathrm{ddd}, J=2.6,5.1,16.9 \mathrm{~Hz}, 1 \mathrm{H})$, $2.61(\mathrm{dd}, J=6.2,16.5 \mathrm{~Hz}, 1 \mathrm{H}), 2.71(\mathrm{dd}, J=4.4,16.5 \mathrm{~Hz}, 1 \mathrm{H}), 4.09(\mathrm{~m}, 1 \mathrm{H}) ;{ }^{13} \mathrm{C} \mathrm{NMR}(75 \mathrm{MHz}) \delta-4.94$, $-4.79,17.88,25.46,25.56 \times 3,27.11,67.23,71.67,79.09,117.35$; IR (neat) 3300, 2930, 2250, $2120 \mathrm{~cm}^{-1}$; HRMS calcd for $\mathrm{C}_{12} \mathrm{H}_{21} \mathrm{NOSi}\left(\mathrm{M}^{+}\right) \mathrm{m} / \mathrm{z} 223.1392$, found 223.1373 .

(3R)-3-(t-Butyldimethylsilyloxy)-5-hexynal (13)

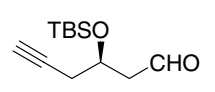

The following reaction was carried out under argon. To a cooled $\left(-52{ }^{\circ} \mathrm{C}\right)$, stirred solution of (3R)-3-(t-butyldimethylsilyloxy)-5-hexynenitrile $(5.49 \mathrm{~g}, 24.6 \mathrm{mmol})$ in toluene $(100 \mathrm{~mL})$ was added Dibal-H ( $35 \mathrm{~mL}$ of $1.04 \mathrm{M}$ solution in toluene, $37 \mathrm{mmol}$ ). The mixture was stirred at $-52{ }^{\circ} \mathrm{C}$ for $2 \mathrm{~h}$ and then quenched with EtOH. This was diluted with $0.2 \mathrm{M}$ aqueous $\mathrm{HCl}(200 \mathrm{~mL})$, and extracted with hexane. The combined organic layers were washed with saturated brine-saturated aqueous $\mathrm{NaHCO}_{3}(1: 1)$. The organic layer was dried and concentrated in vacuo. The residue was purified by column chromatography on silica gel (EtOAc/hexane, 1:40) to provide $5.56 \mathrm{~g}$ (quant.) of $\mathbf{1 3}$ as a colorless oil; TLC, $\mathrm{R}_{f} 0.69$ (EtOAc/hexane, 1:5); $[\alpha]^{21}{ }_{\mathrm{D}}-27.1\left(c 1.11, \mathrm{CHCl}_{3}\right) ;{ }^{1} \mathrm{H}$ NMR $(270 \mathrm{MHz}) \delta 0.08,0.11(2 \mathrm{~s}, 3 \mathrm{H} \times 2), 0.87(\mathrm{~s}, 9 \mathrm{H}), 2.04(\mathrm{t}, J=2.8 \mathrm{~Hz}$, $1 \mathrm{H}), 2.39$ (ddd, $J=2.8,7.2,16.7 \mathrm{~Hz}, 1 \mathrm{H}), 2.47(\mathrm{ddd}, J=2.8,5.0,16.7 \mathrm{~Hz}, 1 \mathrm{H}), 2.67(\mathrm{ddd}, J=2.4,6.8,16.1$ $\mathrm{Hz}, 1 \mathrm{H}), 2.77(\mathrm{ddd}, J=1.7,4.6,16.1 \mathrm{~Hz}, 1 \mathrm{H}), 4.36(\mathrm{~m}, 1 \mathrm{H}), 9.82(\mathrm{dd}, J=1.7,2.4 \mathrm{~Hz}, 1 \mathrm{H}) ;{ }^{13} \mathrm{C} \mathrm{NMR}(68$ MHz) $\delta-4.93,-4.55,17.91,25.63 \times 3,27.64,50.19,66.66,71.10,80.17,201.23$; IR (neat) 3300, 2930, 2120, $1730 \mathrm{~cm}^{-1}$; HRMS calcd for $\mathrm{C}_{8} \mathrm{H}_{13} \mathrm{O}_{2} \mathrm{Si}\left(\mathrm{M}^{+}-t-\mathrm{Bu}\right) \mathrm{m} / \mathrm{z} 169.0685$, found 169.0685 .

(2RS,4R)-4-(t-Butyldimethylsilyloxy)-1-(2,2-dimethyl-1,3-dioxin-4-one-6-yl)-6-heptyn-2-ol (ca. 1:1 diastereomeric mixture)

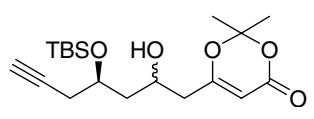

The following reaction was carried out under argon. To a cooled $\left(-78^{\circ} \mathrm{C}\right)$, stirred solution of $\mathbf{1 3}(5.56 \mathrm{~g}, 24.6$ $\mathrm{mmol})$ in $\mathrm{CH}_{2} \mathrm{Cl}_{2}(100 \mathrm{~mL})$ were added $\mathrm{BF}_{3} \cdot \mathrm{Et}_{2} \mathrm{O}(3.3 \mathrm{~mL}, 26 \mathrm{mmol})$ and 2,2-dimethyl-6-methylene-4(trimethylsilyloxy)-1,3-dioxin $(6.90 \mathrm{~g}, 32.2 \mathrm{mmol})$. The mixture was stirred at $-78{ }^{\circ} \mathrm{C}$ for $45 \mathrm{~min}$ and then quenched with saturated aqueous $\mathrm{NaHCO}_{3}$. This was diluted with saturated aqueous $\mathrm{NaHCO}_{3}(200 \mathrm{~mL})$, and extracted with $\mathrm{CH}_{2} \mathrm{Cl}_{2}$. The combined organic layers were dried and concentrated in vacuo. The residue was purified by column chromatography on silica gel (EtOAc/hexane, 1:3) to provide $6.65 \mathrm{~g}(73 \%)$ of the secondary alcohol as a colorless oil; TLC, $\mathrm{R}_{f} 0.28$ (EtOAc/hexane, 1:2); ${ }^{1} \mathrm{H}$ NMR (270 MHz) $\delta 0.12,0.14$, $0.15(3 \mathrm{~s}$, total $6 \mathrm{H}) 0.90,0.91(2 \mathrm{~s}$, total $9 \mathrm{H}), 1.70(\mathrm{~s}, 6 \mathrm{H}), 1.72-2.04(\mathrm{~m}, 3 \mathrm{H}), 2.30-2.48(\mathrm{~m}, 4 \mathrm{H}), 3.05(\mathrm{~d}, J=$ $2.9 \mathrm{~Hz}, 1 \mathrm{H} \times 1 / 2), 3.20(\mathrm{~d}, J=1.1 \mathrm{~Hz}, 1 \mathrm{H} \times 1 / 2), 4.00-4.30(\mathrm{~m}, 2 \mathrm{H}), 5.34,5.35$ (2s, total 1H); IR (neat) 3450, $3300,2930,2120,1720,1635 \mathrm{~cm}^{-1}$; HRMS calcd for $\mathrm{C}_{18} \mathrm{H}_{29} \mathrm{O}_{5} \mathrm{Si}\left(\mathrm{M}^{+}-\mathrm{CH}_{3}\right) \mathrm{m} / \mathrm{z} 353.1784$, found 353.1810. 
(4R)-4-(t-Butyldimethylsilyloxy)-1-(2,2-dimethyl-1,3-dioxin-4-one-6-yl)-6-heptyn-2-one

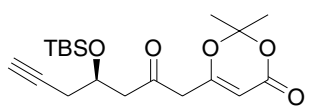

To a cooled $\left(0{ }^{\circ} \mathrm{C}\right)$, stirred solution of $(2 R S, 4 R)-4$-( $t$-butyldimethylsilyloxy)-1-(2,2-dimethyl-1,3-dioxin4-one-6-yl)-6-heptyn-2-ol (12.58 g, $34.13 \mathrm{mmol})$ in $\mathrm{CH}_{2} \mathrm{Cl}_{2}(250 \mathrm{~mL})$ was added Dess-Martin periodinane $(17.4 \mathrm{~g}, 40.9 \mathrm{mmol})$. The mixture was stirred for $1.5 \mathrm{~h}$ and then Dess-Martin periodinane $(33.3 \mathrm{~g}, 78.5 \mathrm{mmol})$ was added at $0{ }^{\circ} \mathrm{C}$. The mixture was stirred for $5 \mathrm{~h}$ and then diluted with saturated aqueous $\mathrm{NaHCO}_{3}-20 \%$ aqueous $\mathrm{Na}_{2} \mathrm{~S}_{2} \mathrm{O}_{3}(1: 1,400 \mathrm{~mL})$ and $\mathrm{CH}_{2} \mathrm{Cl}_{2}(200 \mathrm{~mL})$ at $0{ }^{\circ} \mathrm{C}$. The mixture was stirred for $20 \mathrm{~min}$ and then the organic layer was separated. The aqueous layer was extracted with $\mathrm{CH}_{2} \mathrm{Cl}_{2}$. The combined organic layers were dried and concentrated in vacuo to give the crude ketone, which was used in the next step without purification. In a small-scale experiment, the pure ketone was obtained by column chromatography on silica gel (EtOAc/hexane, 1:10) as a colorless oil; TLC, $\mathrm{R}_{f} 0.40$ (EtOAc/hexane, 1:2); $[\alpha]^{21}{ }_{\mathrm{D}}-46.9\left(c 1.56, \mathrm{CHCl}_{3}\right.$ ); ${ }^{1} \mathrm{H}$ NMR $(270 \mathrm{MHz}) \delta 0.05,0.10(2 \mathrm{~s}, 3 \mathrm{H} \times 2), 0.87(\mathrm{~s}, 9 \mathrm{H}), 1.72(\mathrm{~s}, 6 \mathrm{H}), 2.03(\mathrm{t}, J=2.7 \mathrm{~Hz}, 1 \mathrm{H}), 2.34(\mathrm{ddd}, J$ $=2.7,7.0,16.7 \mathrm{~Hz}, 1 \mathrm{H}), 2.42(\mathrm{ddd}, J=2.7,4.8,16.7 \mathrm{~Hz}, 1 \mathrm{H}), 2.78(\mathrm{~m}, 2 \mathrm{H}), 3.34(\mathrm{~d}, J=16.9 \mathrm{~Hz}, 1 \mathrm{H}), 3.41$ $(\mathrm{d}, J=16.9 \mathrm{~Hz}, 1 \mathrm{H}), 4.31(\mathrm{~m}, 1 \mathrm{H}), 5.33(\mathrm{~s}, 1 \mathrm{H}) ;{ }^{13} \mathrm{C} \mathrm{NMR}(68 \mathrm{MHz}) \delta-4.96,-4.73,17.91,24.96,25.05$, $25.68 \times 3,27.36,48.64,49.18,67.24,71.13,80.08,96.73,107.21,160.68,164.25,201.69$; IR (neat) 3300, 2930, 2120, 1730, $1640 \mathrm{~cm}^{-1}$; HRMS calcd for $\mathrm{C}_{15} \mathrm{H}_{21} \mathrm{O}_{5} \mathrm{Si}\left(\mathrm{M}^{+}-t-\mathrm{Bu}\right) \mathrm{m} / \mathrm{z}$ 309.1158, found 309.1169 .

(4R)-1-(2,2-Dimethyl-1,3-dioxin-4-one-6-yl)-4-hydroxy-6-heptyn-2-one (15)

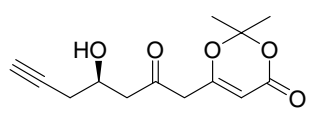

To a cooled $\left(0{ }^{\circ} \mathrm{C}\right)$, stirred solution of the crude ketone obtained above in MeCN (250 mL) was added $48 \%$ aq. $\mathrm{HF}(12 \mathrm{~mL})$. The mixture was stirred at room temperature for $8 \mathrm{~h}$ and then quenched with saturated aqueous $\mathrm{NaHCO}_{3}$. This was diluted with saturated aqueous $\mathrm{NaHCO}_{3}(400 \mathrm{~mL})$, and extracted with $\mathrm{CH}_{2} \mathrm{Cl}_{2}$. The combined organic layers were dried and concentrated in vacuo. The residue was purified by column chromatography on silica gel (EtOAc/hexane, 1:2) to provide $\mathbf{1 5}$ (7.95 g, 92\%, 2 steps) as a colorless oil; TLC, $\mathrm{R}_{f} 0.15$ (EtOAc/hexane, 1:1); $[\alpha]^{22}{ }_{\mathrm{D}}-38.3\left(c\right.$ 1.21, $\left.\mathrm{CHCl}_{3}\right) ;{ }^{1} \mathrm{H}$ NMR $(270 \mathrm{MHz}) \delta 1.72(\mathrm{~s}, 6 \mathrm{H}), 2.09$ (t, $J=2.6 \mathrm{~Hz}, 1 \mathrm{H}), 2.42(\mathrm{ddd}, J=2.6,6.6,16.9 \mathrm{~Hz}, 1 \mathrm{H}), 2.49$ (ddd, $J=2.6,5.9,16.9 \mathrm{~Hz}, 1 \mathrm{H}), 2.77$ (dd, $J=8.1$, $17.6 \mathrm{~Hz}, 1 \mathrm{H}), 2.87$ (dd, $J=3.7,17.6 \mathrm{~Hz}, 1 \mathrm{H}), 2.93(\mathrm{~d}, J=4.4 \mathrm{~Hz}, 1 \mathrm{H}), 3.41(\mathrm{~s}, 2 \mathrm{H}), 4.25(\mathrm{~m}, 1 \mathrm{H}), 5.37$ (s, $1 \mathrm{H}) ;{ }^{13} \mathrm{C}$ NMR $(68 \mathrm{MHz}) \delta 24.96 \times 2,26.26,47.74,48.09,65.77,71.41,79.71,96.87,107.27,160.57,163.82$, 203.16; IR (neat) 3430, 3300, 2920, 2120, 1720, $1640 \mathrm{~cm}^{-1}$; HRMS calcd for $\mathrm{C}_{13} \mathrm{H}_{17} \mathrm{O}_{5}\left(\mathrm{M}^{+}+\mathrm{H}\right) \mathrm{m} / \mathrm{z} 253.1076$, found 253.1075 .

(2R,4R)-1-(2,2-Dimethyl-1,3-dioxin-4-one-6-yl)-6-heptyn-2,4-diol

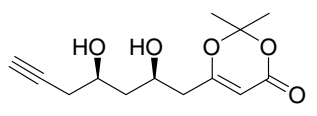

The following reaction was carried out under argon. To a cooled $\left(-78{ }^{\circ} \mathrm{C}\right)$, stirred solution of $\mathbf{1 5}(7.95 \mathrm{~g}, 31.5$ mmol) in THF-MeOH $(150 \mathrm{~mL})$ was added $\mathrm{Et}_{2} \mathrm{BOMe}(38 \mathrm{~mL}$ of $1.0 \mathrm{M}$ solution in THF, $38 \mathrm{mmol})$. The 
mixture was stirred at $-78{ }^{\circ} \mathrm{C}$ for $30 \mathrm{~min}$ and then $\mathrm{NaBH}_{4}(1.70 \mathrm{~g}, 44.9 \mathrm{mmol})$ was added. The mixture was stirred at $-78{ }^{\circ} \mathrm{C}$ for $8 \mathrm{~h}$. This was quenched with $\mathrm{AcOH}$ and diluted with saturated aqueous $\mathrm{NaHCO}_{3}(300$ $\mathrm{mL}$ ), and extracted with EtOAc. The combined organic layers were dried and concentrated in vacuo. The residue was purified by column chromatography on silica gel (EtOAc/hexane, 2:3) to provide $8.01 \mathrm{~g}$ (quant.) of the diol as a colorless oil; TLC, $\mathrm{R}_{f} 0.10$ (EtOAc/hexane, 1:1); $[\alpha]^{22}{ }_{\mathrm{D}}-14.8\left(c\right.$ 1.11, $\left.\mathrm{CHCl}_{3}\right) ;{ }^{1} \mathrm{H}$ NMR $(270$ MHz) $\delta 1.71(\mathrm{~s}, 6 \mathrm{H}), 1.60-1.86(\mathrm{~m}, 2 \mathrm{H}), 2.09$ (t, $J=2.6 \mathrm{~Hz}, 1 \mathrm{H}), 2.34-2.49(\mathrm{~m}, 4 \mathrm{H}), 3.29$ (brd, $J=10.6 \mathrm{~Hz}$, 1H), 3.68 (brd, $J=7.7 \mathrm{~Hz}, 1 \mathrm{H}), 4.05(\mathrm{~m}, 1 \mathrm{H}), 4.18(\mathrm{~m}, 1 \mathrm{H}), 5.35(\mathrm{~s}, 1 \mathrm{H}) ;{ }^{13} \mathrm{C}$ NMR $(68 \mathrm{MHz}) \delta 24.82,25.20$, 27.79, 41.47, 41.90, 69.00, 70.55, 71.27, 80.05, 95.20, 106.69, 161,26, 168.72; IR (neat) 3410, 3300, 2910, 2120, 1720, $1640 \mathrm{~cm}^{-1}$; HRMS calcd for $\mathrm{C}_{13} \mathrm{H}_{19} \mathrm{O}_{5}\left(\mathrm{M}^{+}+\mathrm{H}\right) \mathrm{m} / \mathrm{z} 255.1233$, found 255.1234 .

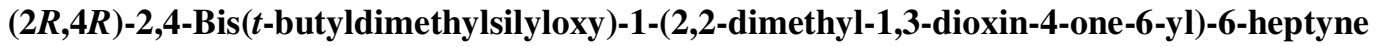

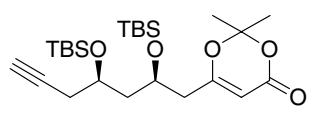

To a cooled $\left(0{ }^{\circ} \mathrm{C}\right)$, stirred solution of $(2 R, 4 R)$-1-(2,2-dimethyl-1,3-dioxin-4-one-6-yl)-6-heptyn-2, 4-diol (4.87 g, $19.2 \mathrm{mmol})$ in DMF (40 mL) were added imidazole $(5.49 \mathrm{~g}, 80.6 \mathrm{mmol})$ and TBSCl (6.06 g, $40.2 \mathrm{mmol})$. The mixture was stirred for $5 \mathrm{~h}$ and then diluted with saturated aqueous $\mathrm{NaHCO}_{3}(250 \mathrm{~mL})$. This was extracted with $\mathrm{CH}_{2} \mathrm{Cl}_{2}$. The combined organic layers were dried and concentrated in vacuo. The residue was purified by column chromatography on silica gel (EtOAc/hexane, 1:10) to provide $8.58 \mathrm{~g}(93 \%)$ of the bisTBS ether as a colorless oil; TLC, $\mathrm{R}_{f} 0.48$ (EtOAc/toluene, 1:10); $[\alpha]^{22}{ }_{\mathrm{D}}-40.9\left(c 1.58, \mathrm{CHCl}_{3}\right) ;{ }^{1} \mathrm{H} \mathrm{NMR}$ $(270 \mathrm{MHz}) \delta 0.05,0.07,0.09,0.10(4 \mathrm{~s}, 3 \mathrm{H} \times 4), 0.88,0.90(2 \mathrm{~s}, 9 \mathrm{H} \times 2), 1.69 \times 2(2 \mathrm{~s}, 3 \mathrm{H} \times 2), 1.79(\mathrm{ddd}, J=$ $5.1,7.7,13.9 \mathrm{~Hz}, 1 \mathrm{H}), 1.88(\mathrm{ddd}, J=4.4,8.1,13.9 \mathrm{~Hz}, 1 \mathrm{H}), 2.00(\mathrm{t}, J=2.6 \mathrm{~Hz}, 1 \mathrm{H}), 2.31(\mathrm{dd}, J=7.3,13.6$ $\mathrm{Hz}, 1 \mathrm{H}), 2.34(\mathrm{~m}, 2 \mathrm{H}), 2.44(\mathrm{dd}, J=4.2,13.6 \mathrm{~Hz}, 1 \mathrm{H}), 3.86(\mathrm{~m}, 1 \mathrm{H}), 4.13(\mathrm{~m}, 1 \mathrm{H}), 5.29(\mathrm{~s}, 1 \mathrm{H}) ;{ }^{13} \mathrm{C} \mathrm{NMR}$ $(68 \mathrm{MHz}) \delta ;-4.73,-4.44,-4.29 \times 2,17.94 \times 2,24.30,25.74 \times 6,25.89,27.70,41.58,44.26,66.46,67.93$, 70.64, 80.74, 95.66, 106.32, 161.00, 169.12; IR (neat) 3310, 2930, 2120, 1730, $1640 \mathrm{~cm}^{-1}$; HRMS calcd for $\mathrm{C}_{21} \mathrm{H}_{37} \mathrm{O}_{5} \mathrm{Si}_{2}\left(\mathrm{M}^{+}-t-\mathrm{Bu}\right) \mathrm{m} / \mathrm{z} 425.2180$, found 425.2191 .

(2R,4R)-7-Bromo-2,4-bis(t-butyldimethylsilyloxy)-1-(2,2-dimethyl-1,3-dioxin-4-one-6-yl)-6-heptyne (16)

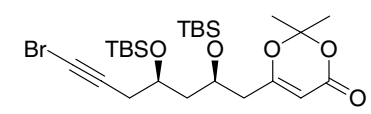

To a cooled $\left(0{ }^{\circ} \mathrm{C}\right)$, stirred solution of $(2 R, 4 R)$-2,4-bis( $t$-butyldimethylsilyloxy)-1-(2,2-dimethyl-1,3-dioxin4-one-6-yl)-6-heptyne $(8.58 \mathrm{~g}, 17.8 \mathrm{mmol})$ in acetone $(180 \mathrm{~mL})$ were added $\mathrm{AgNO}_{3}(0.30 \mathrm{~g}, 1.8 \mathrm{mmol})$ and NBS (3.81 g, $21.4 \mathrm{mmol})$. The mixture was stirred at room temperature for $30 \mathrm{~min}$ and then diluted with saturated aqueous $\mathrm{NaHCO}_{3}(200 \mathrm{~mL})$. This was extracted with $\mathrm{CH}_{2} \mathrm{Cl}_{2}$. The combined organic layers were dried and concentrated in vacuo. The residue was purified by column chromatography on silica gel (EtOAc/hexane, 1:8) to provide $9.72 \mathrm{~g}(97 \%)$ of $\mathbf{1 6}$ as a colorless oil; TLC, $\mathbf{R}_{f} 0.52$ (EtOAc/toluene, 1:10); $[\alpha]^{21}{ }_{\mathrm{D}}-36.3\left(c 1.38, \mathrm{CHCl}_{3}\right) ;{ }^{1} \mathrm{H} \mathrm{NMR}(300 \mathrm{MHz}) \delta 0.05,0.08 \times 2,0.10(4 \mathrm{~s}, 3 \mathrm{H} \times 4), 0.88,0.90(2 \mathrm{~s}, 9 \mathrm{H} \times 2)$, $1.69,1.70(2 \mathrm{~s}, 3 \mathrm{H} \times 2), 1.80(\mathrm{~m}, 2 \mathrm{H}), 2.32(\mathrm{dd}, J=7.1,13.9 \mathrm{~Hz}, 1 \mathrm{H}), 2.36(\mathrm{~m}, 2 \mathrm{H}), 2.44(\mathrm{dd}, J=4.4,13.9 \mathrm{~Hz}$, 
$1 \mathrm{H}), 3.87(\mathrm{~m}, 1 \mathrm{H}), 4.13(\mathrm{~m}, 1 \mathrm{H}), 5.29(\mathrm{~s}, 1 \mathrm{H}),{ }^{13} \mathrm{C}$ NMR $(75 \mathrm{MHz}) \delta-4.77,-4.71,-4.49,-4.36,17.91 \times 2$, 24.28, $25.73 \times 6,25.92,28.84,40.28,41.63,44.50,66.42,67.77,76.84,95.65,106.35,160.98,169.02$; IR (neat) $2930,1730,1640 \mathrm{~cm}^{-1}$; HRMS calcd for $\mathrm{C}_{21} \mathrm{H}_{36} \mathrm{O}_{5} \mathrm{Si}_{2} \mathrm{Br}^{79}\left(\mathrm{M}^{+}-t-\mathrm{Bu}\right) \mathrm{m} / \mathrm{z}$ 503.1284, found 503.1288.

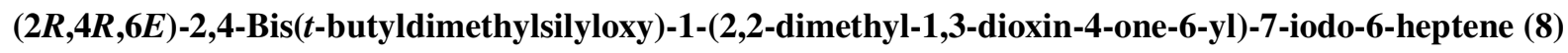

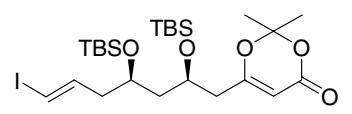

The following reaction was carried out under argon. To a stirred solution of $\mathbf{1 6}$ (9.72 g, $17.3 \mathrm{mmol})$ in argon-bubbled THF $(200 \mathrm{~mL})$ were added $\mathrm{Pd}_{2}(\mathrm{dba})_{3}(79.2 \mathrm{mg}, 0.0865 \mathrm{mmol})$ and $\mathrm{PPh}_{3}(181.5 \mathrm{mg}, 0.692$ mmol). Then a solution of $\mathrm{Bu}_{3} \mathrm{SnH}(14 \mathrm{~mL}, 52 \mathrm{mmol})$ in THF $(50 \mathrm{~mL})$ was slowly added over $20 \mathrm{~min}$. The mixture was stirred for $2 \mathrm{~h}$ and then concentrated in vacuo. The residue was dissolved in $\mathrm{CH}_{2} \mathrm{Cl}_{2}(200 \mathrm{~mL})$ and stirred at $0{ }^{\circ} \mathrm{C}$. To the solution $\mathrm{I}_{2}(9.66 \mathrm{~g}, 38.1 \mathrm{mmol})$ was added. The mixture was stirred for $1 \mathrm{~h}$ and then diluted with $10 \%$ aqueous $\mathrm{Na}_{2} \mathrm{~S}_{2} \mathrm{O}_{3}(200 \mathrm{~mL})$. This was extracted with $\mathrm{CH}_{2} \mathrm{Cl}_{2}$. The combined organic layers were dried and concentrated in vacuo. The residue was purified by column chromatography on silica gel (EtOAc/hexane, 1:20) to provide $8.82 \mathrm{~g}(84 \%)$ of $\mathbf{8}$ as a colorless oil; TLC, $\mathrm{R}_{f} 0.29$ (EtOAc/hexane, 1:5); $[\alpha]^{21}-16.4\left(c 1.58, \mathrm{CHCl}_{3}\right) ;{ }^{1} \mathrm{H}$ NMR $(270 \mathrm{MHz}) \delta 0.05,0.06,0.07(3 \mathrm{~s}$, total $12 \mathrm{H}), 0.88,0.90(2 \mathrm{~s}, 9 \mathrm{H} \times 2)$, $1.58(\mathrm{~m}, 1 \mathrm{H}), 1.68,1.70(2 \mathrm{~s}, 3 \mathrm{H} \times 2), 1.73(\mathrm{~m}, 1 \mathrm{H}), 2.21(\mathrm{~m}, 2 \mathrm{H}), 2.30(\mathrm{dd}, J=6.8,13.9 \mathrm{~Hz}, 1 \mathrm{H}), 2.40(\mathrm{dd}, J=$ 4.9, $13.9 \mathrm{~Hz}, 1 \mathrm{H}), 3.79(\mathrm{~m}, 1 \mathrm{H}), 4.05(\mathrm{~m}, 1 \mathrm{H}), 5.27(\mathrm{~s}, 1 \mathrm{H}), 6.06(\mathrm{~d}, J=14.3 \mathrm{~Hz}, 1 \mathrm{H}), 6.48(\mathrm{dt}, J=14.3,7.4$ $\mathrm{Hz}, 1 \mathrm{H}) ;{ }^{13} \mathrm{C}$ NMR $(68 \mathrm{MHz})-4.58 \times 2,-4.44,-4.21,17.94 \times 2,24.42,25.74 \times 3,25.80 \times 3,25.89,41.84$, 43.86, 44.66, 66.49, 67.96 , 77.14, 95.66, 106.37, 142.34, 160.91, 168.86; IR (neat) 2930, 1730, $1640 \mathrm{~cm}^{-1}$; HRMS calcd for $\mathrm{C}_{21} \mathrm{H}_{38} \mathrm{O}_{5} \mathrm{Si}_{2} \mathrm{I}\left(\mathrm{M}^{+}-t-\mathrm{Bu}\right) \mathrm{m} / \mathrm{z} 553.1303$, found 553.1297 .

$(2 E, 5 R, 6 S, 7 Z, 9 E, 12 R, 14 R)-12,14-B i s(t$-butyldimethylsilyloxy)-1-(t-butyldiphenylsilyloxy)-15-(2,2-dimet hyl-1,3-dioxin-4-one-6-yl)-6-[(4-methoxyphenyl)methoxy]-5-methyl-2,7,9-pentadecatriene

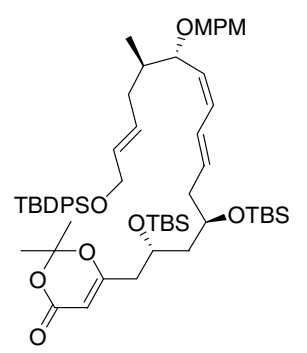

The following reaction was carried out under argon. To a stirred solution of 7 (1.69 g, $2.10 \mathrm{mmol})$ and $\mathbf{8}$ (1.06 $\mathrm{g}, 1.74 \mathrm{mmol})$ in degassed DMSO-THF $(1: 1,40 \mathrm{~mL})$ was added $\mathrm{CuCl}(254 \mathrm{mg}, 2.57 \mathrm{mmol})$. Then a solution of $\mathrm{Pd}\left(\mathrm{PPh}_{3}\right)_{4}(101 \mathrm{mg}, 0.0877 \mathrm{mmol})$ in degassed THF $(3 \mathrm{~mL})$ was added. The mixture was stirred for $1 \mathrm{~h}$ and then $\mathrm{Pd}\left(\mathrm{PPh}_{3}\right)_{4}(10.5 \mathrm{mg}, 0.00909 \mathrm{mmol})$ was added. The mixture was stirred for $1 \mathrm{~h}$ and then diluted with saturated brine-saturated aqueous $\mathrm{NaHCO}_{3}(1: 1,300 \mathrm{~mL})$. This was extracted with $\mathrm{Et}_{2} \mathrm{O}$. The combined organic layers were dried and concentrated in vacuo. The residue was purified by column chromatography on silica gel (EtOAc/hexane, 1:10) to provide $1.68 \mathrm{~g}(97 \%)$ of the triene as a colorless oil; TLC, $\mathrm{R}_{f} 0.57$ 
(EtOAc/hexane, 1:3); $[\alpha]^{21}{ }_{\mathrm{D}}-25.9\left(c 1.36, \mathrm{CHCl}_{3}\right) ;{ }^{1} \mathrm{H}-\mathrm{NMR}(300 \mathrm{MHz}) \delta 0.04 \times 2,0.06(3 \mathrm{~s}$, total $12 \mathrm{H}), 0.86$, $0.89(2 \mathrm{~s}, 9 \mathrm{H} \times 2), 0.93(\mathrm{~d}, J=6.3 \mathrm{~Hz}, 3 \mathrm{H}), 1.04(\mathrm{~s}, 9 \mathrm{H}), 1.66,1.68(2 \mathrm{~s}, 3 \mathrm{H} \times 2), 1.59-1.79(\mathrm{~m}, 4 \mathrm{H}), 2.17-2.34$ (m, 4H), $2.41(\mathrm{dd}, J=4.3,13.8 \mathrm{~Hz}, 1 \mathrm{H}) 3.79(\mathrm{~s}, 3 \mathrm{H}), 3.79(\mathrm{~m}, 1 \mathrm{H}), 4.01(\mathrm{dd}, J=5.9,9.3 \mathrm{~Hz}, 1 \mathrm{H}), 4.09(\mathrm{~m}$, $1 \mathrm{H}), 4.14(\mathrm{~d}, J=4.2 \mathrm{~Hz}, 2 \mathrm{H}), 4.21,4.49(2 \mathrm{~d}, J=11.5 \mathrm{~Hz}, 1 \mathrm{H} \times 2), 5.27(\mathrm{~s}, 1 \mathrm{H}), 5.30(\mathrm{t}, J=9.3 \mathrm{~Hz}, 1 \mathrm{H})$, 5.47-5.72 (m, 3H), 618-6.29 (m, 2H), $6.85(\mathrm{~m}, 2 \mathrm{H}), 7.23(\mathrm{~m}, 2 \mathrm{H}), 7.33-7.41(\mathrm{~m}, 6 \mathrm{H}), 7.66-7.69(\mathrm{~m}, 4 \mathrm{H})$; ${ }^{13} \mathrm{C}-\mathrm{NMR}(75 \mathrm{MHz}) \delta-4.71,-4.59,-4.43,-4.08,15.15,17.88,17.95,19.16,24.30,25.71 \times 3,25.81 \times 4$, $26.78 \times 3,35.42$, 38.46, 41.12, 41.68, 44.87, 55.15, 64.47, 66.60, 68.96, 69.58, 77.59, 95.60, 106.26, 113.57×2, $127.55 \times 4,128.06,129.20 \times 2,129.43,129.51 \times 3,130.25,130.96,131.96,132.06,133.82 \times 2,135.46 \times 4,158.94$, 160.89, 169.04; IR (neat) 2930, 1730, 1640, 1615, $1515 \mathrm{~cm}^{-1}$; HRMS (FAB) calcd for $\mathrm{C}_{55} \mathrm{H}_{83} \mathrm{O}_{7} \mathrm{Si}_{3}$ $\left(\mathrm{M}^{+}\right.$-acetone $\left.+\mathrm{H}\right) \mathrm{m} / \mathrm{z}$ 939.5447, found 939.5452 .

\section{$(2 E, 5 R, 6 S, 7 Z, 9 E, 12 R, 14 R)-12,14-B i s(t$-butyldimethylsilyloxy)-15-(2,2-dimethyl-1,3-dioxin-4-one-6-yl)-6} -[(4-methoxyphenyl)methoxy]-5-methyl-2,7,9-pentadecatrien-1-ol (17)

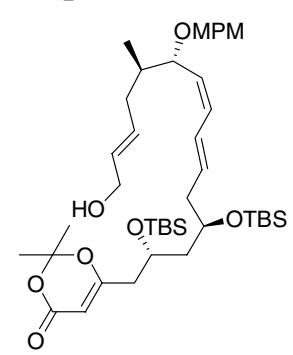

To a cooled $\left(0{ }^{\circ} \mathrm{C}\right)$, stirred solution of $(2 E, 5 R, 6 S, 7 Z, 9 E, 12 R, 14 R)$-12,14-bis( $t$-butyldimethylsilyloxy)-1( $t$-butyldiphenylsilyloxy)-15-(2,2-dimethyl-1,3-dioxin-4-one-6-yl)-6-[(4-methoxyphenyl)methoxy]-5-methyl2,7,9-pentadecatriene $(1.68 \mathrm{~g}, 1.68 \mathrm{mmol})$ in $\mathrm{MeOH}(35 \mathrm{~mL})$ was added $\mathrm{NH}_{4} \mathrm{~F}(351 \mathrm{mg}, 9.48 \mathrm{mmol})$. The mixture was stirred for $14 \mathrm{~h}$ and then $\mathrm{NH}_{4} \mathrm{~F}(35.5 \mathrm{mg}, 0.958 \mathrm{mmol})$ was added. The mixture was stirred for $10 \mathrm{~h}$ and quenched with saturated aqueous $\mathrm{NaHCO}_{3}$. This was diluted with saturated aqueous $\mathrm{NaHCO}_{3}(200$ $\mathrm{mL}$ ), and extracted with $\mathrm{CH}_{2} \mathrm{Cl}_{2}$. The combined organic layers were dried and concentrated in vacuo. The residue was purified by column chromatography on silica gel (EtOAc/hexane, 1:8 to 1:3) to provide $1.20 \mathrm{~g}$ (94\%) of 17 as a colorless oil, and $28.8 \mathrm{mg}(2 \%)$ of the TBDPS ether was recovered; TLC, $\mathrm{R}_{f} 0.53$ (EtOAc/hexane, 1:1); $[\alpha]^{20}{ }_{\mathrm{D}}-22.8\left(c 1.17, \mathrm{CHCl}_{3}\right) ;{ }^{1} \mathrm{H}-\mathrm{NMR}(300 \mathrm{MHz}) \delta 0.04,0.05,0.07(3 \mathrm{~s}$, total $12 \mathrm{H})$, 0.86, $0.90(2 \mathrm{~s}, 9 \mathrm{H} \times 2), 0.94(\mathrm{~d}, J=6.6 \mathrm{~Hz}, 3 \mathrm{H}), 1.67,1.68(2 \mathrm{~s}, 3 \mathrm{H} \times 2), 1.55-1.81(\mathrm{~m}, 4 \mathrm{H}), 2.23-2.32(\mathrm{~m}, 4 \mathrm{H})$, $2.41(\mathrm{dd}, J=4.2,13.8 \mathrm{~Hz}, 1 \mathrm{H}), 3.80$ (s, 3H), $3.80(\mathrm{~m}, 1 \mathrm{H}), 3.99-4.09$ (m, 4H), 4.21, 4.49 (2d, $J=11.6 \mathrm{~Hz}$, $1 \mathrm{H} \times 2), 5.27(\mathrm{~s}, 1 \mathrm{H}), 5.30(\mathrm{t}, J=9.5 \mathrm{~Hz}, 1 \mathrm{H}), 5.52-5.73(\mathrm{~m}, 3 \mathrm{H}), 6.18-6.28(\mathrm{~m}, 2 \mathrm{H}), 6.86(\mathrm{~m}, 2 \mathrm{H}), 7.23(\mathrm{~m}$, $2 \mathrm{H}) ;{ }^{13} \mathrm{C}-\mathrm{NMR}(68 \mathrm{MHz}) \delta-4.70,-4.58,-4.44,-4.09,15.20,17.88,17.97,24.33,25.71 \times 4,25.83 \times 3,35.39$, $38.33,41.18,41.70,44.86,55.20,63.61,66.63,68.97,69.57,77.32,95.57,106.32,113.57 \times 2,128.06$, $129.27 \times 2,129.41,130.50,130.91,131.37,132.06,132.12,158.96,160.97,169.06$; IR (neat) 3500, 2930, 1730, 1640, 1615, $1515 \mathrm{~cm}^{-1}$; HRMS calcd for $\mathrm{C}_{39} \mathrm{H}_{64} \mathrm{O}_{7} \mathrm{Si}_{2}\left(\mathrm{M}^{+}\right.$-acetone) $\mathrm{m} / \mathrm{z} 700.4191$, found 700.4191 . 
$(2 E, 5 R, 6 S, 7 Z, 9 E, 12 R, 14 R)-12,14-B i s(t$-butyldimethylsilyloxy)-15-(2,2-dimethyl-1,3-dioxin-4-one-6-yl)-1 -methoxycarbonyloxy-6-[(4-methoxyphenyl)methoxy]-5-methyl-2,7,9-pentadecatriene

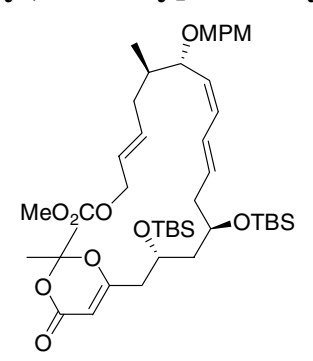

To a cooled $\left(0{ }^{\circ} \mathrm{C}\right)$, stirred solution of $\mathbf{1 7}(1.21 \mathrm{~g}, 1.59 \mathrm{mmol})$ in $\mathrm{CH}_{2} \mathrm{Cl}_{2}(24 \mathrm{~mL})$ were added pyridine $(0.70$ $\mathrm{mL}, 9.7 \mathrm{mmol})$ and methyl chloroformate $(0.40 \mathrm{~mL}, 5.1 \mathrm{mmol})$. The mixture was stirred for $2 \mathrm{~h}$, and this was concentrated in vacuo. The residue was purified by column chromatography on silica gel $\left(\mathrm{Et}_{3} \mathrm{~N} / \mathrm{hexane}, 1: 100\right.$ to EtOAc/hexane, 1:5) to provide $1.29 \mathrm{~g}$ (quant.) of the carbonate as a colorless oil; TLC, $\mathrm{R}_{f} 0.63$ (EtOAc/hexane, 1:1); $[\alpha]^{25}-23.9\left(c 1.77, \mathrm{CHCl}_{3}\right) ;{ }^{1} \mathrm{H}-\mathrm{NMR}(270 \mathrm{MHz}) \delta 0.04,0.05,0.07$ (3s, total 12H), 0.86, $0.90(2 \mathrm{~s}, 9 \mathrm{H} \times 2), 0.92(\mathrm{~d}, J=6.6 \mathrm{~Hz}, 3 \mathrm{H}), 1.67,1.68(2 \mathrm{~s}, 3 \mathrm{H} \times 2), 1.54-1.83(\mathrm{~m}, 4 \mathrm{H}), 2.17-2.32(\mathrm{~m}, 4 \mathrm{H})$, $2.43(\mathrm{dd}, J=4.4,13.9 \mathrm{~Hz}, 1 \mathrm{H}), 3.77(\mathrm{~s}, 3 \mathrm{H}), 3.77(\mathrm{~m}, 1 \mathrm{H}), 3.80(\mathrm{~s}, 3 \mathrm{H}), 4.02(\mathrm{dd}, J=5.7,9.5 \mathrm{~Hz}, 1 \mathrm{H}), 4.10$ $(\mathrm{m}, 1 \mathrm{H}), 4.20,4.49(2 \mathrm{~d}, J=11.7 \mathrm{~Hz}, 1 \mathrm{H} \times 2), 4.54(\mathrm{~d}, J=6.2 \mathrm{~Hz}, 2 \mathrm{H}), 5.27(\mathrm{~s}, 1 \mathrm{H}), 5.28(\mathrm{t}, J=9.5 \mathrm{~Hz}, 1 \mathrm{H})$, $5.53(\mathrm{dt}, J=15.0,6.2 \mathrm{~Hz}, 1 \mathrm{H}), 5.63-5.78(\mathrm{~m}, 2 \mathrm{H}), 6.17-6.25(\mathrm{~m}, 2 \mathrm{H}), 6.86(\mathrm{~m}, 2 \mathrm{H}), 7.22(\mathrm{~m}, 2 \mathrm{H}) ;{ }^{13} \mathrm{C}-\mathrm{NMR}$ $(68 \mathrm{MHz}) \delta-4.67,-4.55,-4.41,-4.06,15.15,17.91,18.00,24.36,25.74 \times 3,25.83 \times 4,35.45,38.21,41.18$, $41.75,44.92,54.65,55.23,66.63,68.53,69.00,69.60,77.29,95.63,106.32,113.63 \times 2,124.69,127.97$, 129.27×3, 130.88, 132.23×2, 135.75, 155.64, 158.98, 160.97, 169.09; IR (neat) 2930, 1750, 1730, 1640, 1615, $1515 \mathrm{~cm}^{-1}$ : HRMS calcd for $\mathrm{C}_{40} \mathrm{H}_{63} \mathrm{O}_{10} \mathrm{Si}_{2}\left(\mathrm{M}^{+}-t-\mathrm{Bu}\right) \mathrm{m} / \mathrm{z} 759.3960$, found 759.3974 .

Methyl (5R,7R,9E,11Z,13S,14R,16E)-5,7-Bis(t-butyldimethylsilyloxy)-18-(methoxycarbonyl)oxy-13-[(4methoxyphenyl)methoxy]-14-methyl-3-oxo-9,11,16-octadecatriene-1-carboxylate (6)

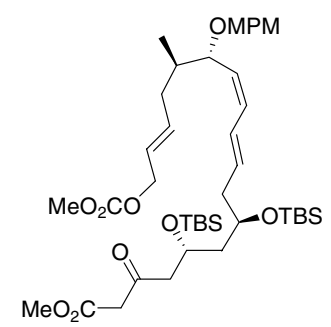

(2E,5R,6S,7Z,9E,12R,14R)-12,14-bis(t-butyldimethylsilyloxy)-15-(2,2-dimethyl-1,3-dioxin-4-one-6-yl)-1-me thoxycarbonyloxy-6-[(4-methoxyphenyl)methoxy]-5-methyl-2,7,9-pentadecatriene (1.29 g $1.59 \mathrm{mmol})$ was dissolved in degassed toluene- $\mathrm{MeOH}(8: 1,25 \mathrm{~mL})$. The solution was transferred into three $20 \mathrm{~mL}$ sealed tubes equipped with a screwed stopper, and the tubes were filled with argon. The tubes were heated to $110{ }^{\circ} \mathrm{C}$ for $3 \mathrm{~h}$. After being cooled to ambient temperature, the solution was concentrated in vacuo. The residue was purified by column chromatography on silica gel (EtOAc/hexane, 1:5) to provide $1.23 \mathrm{~g}(98 \%)$ of 6 as a colorless oil; TLC, $\mathrm{R}_{f} 0.74$ (EtOAc/hexane, 1:2); $[\alpha]^{26}{ }_{\mathrm{D}}-22.3\left(c\right.$ 1.11, $\left.\mathrm{CHCl}_{3}\right) ;{ }^{1} \mathrm{H}-\mathrm{NMR}(270 \mathrm{MHz}) \delta 0.03$, 
0.05, $0.06(3 \mathrm{~s}$, total $12 \mathrm{H}), 0.86,0.89(2 \mathrm{~s}, 9 \mathrm{H} \times 2), 0.92(\mathrm{~d}, J=6.7 \mathrm{~Hz}, 3 \mathrm{H}), 1.52-1.86(\mathrm{~m}, 4 \mathrm{H}), 2.17-2.37(\mathrm{~m}$, $3 \mathrm{H}+2 \mathrm{H} \times 1 / 7$, enol form), 2.67-2.69 (m, $2 \mathrm{H} \times 6 / 7$, keto form), 3.48 (s, $2 \mathrm{H} \times 6 / 7$, keto form), 3.72 (s, $3 \mathrm{H}$ ), 3.77 (s, $3 \mathrm{H}), 3.78(\mathrm{~m}, 1 \mathrm{H}), 3.80(\mathrm{~s}, 3 \mathrm{H}), 4.01(\mathrm{dd}, J=5.5,9.2 \mathrm{~Hz}, 1 \mathrm{H}), 4.21,4.49(2 \mathrm{~d}, J=11.6 \mathrm{~Hz}, 1 \mathrm{H} \times 2), 4.28(\mathrm{~m}$, $1 \mathrm{H}), 4.55(\mathrm{~d}, J=6.1 \mathrm{~Hz}, 2 \mathrm{H}), 5.01(\mathrm{~s}, 1 \mathrm{H} \times 1 / 7$, enol form), 5.29 (t, $J=9.2 \mathrm{~Hz}, 1 \mathrm{H}), 5.51$ (dt, $J=15.36 .1 \mathrm{~Hz}$, $1 \mathrm{H})$, 5.65-5.79 (m, 2H), 6.21-6.25 (m, $2 \mathrm{H}), 6.86(\mathrm{~m}, 2 \mathrm{H}), 7.23(\mathrm{~m}, 2 \mathrm{H}), 11.94$ (s, $1 \mathrm{H} \times 1 / 7$, enol form); ${ }^{13} \mathrm{C}-\mathrm{NMR}$ (68 MHz, keto form) $\delta-4.81,-4.52 \times 2,-4.21,15.17,17.88,17.97,25.83 \times 6,35.48,38.21,40.92$, $44.78,50.05,50.68,52.21,54.65,55.23,66.49,68.56,68.91,69.56,77.29,113.60 \times 2,124.63,127.86,129.01$, $129.30 \times 2,130.97,132.43,132.55,135.80,155.64,158.98,167.45,201.60$ (derived from enol form $\delta 43.57$, 45.09, 51.08, 67.01, 91.17, 12.74, 128.92, 132.78, 172.81, 175.66); IR (neat) 2930, 1750, 1615, $1515 \mathrm{~cm}^{-1}$; HRMS (FAB) calcd for $\mathrm{C}_{42} \mathrm{H}_{70} \mathrm{O}_{10} \mathrm{Si}_{2} \mathrm{Na}\left(\mathrm{M}^{+}+\mathrm{Na}\right) \mathrm{m} / \mathrm{z} 813.4406$, found 813.4416.

$(2 R S, 4 E, 7 R, 8 S, 9 Z, 11 E, 14 R, 16 R)-14,16-B i s(t$-butyldimethylsilyloxy)-2-methoxycarbonyl-8-[(4-methoxyphenyl)methoxy]-7-methyl-4,9,11-cycloheptadecatrien-1-one (18, ca. 3:2 diastereomeric mixture)

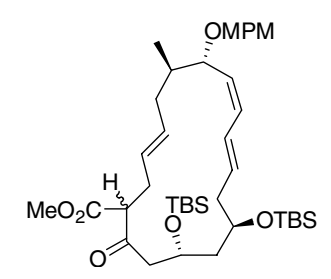

To a stirred solution of $\mathrm{Pd}\left(\mathrm{PPh}_{3}\right)_{4}(113 \mathrm{mg}, 0.0976 \mathrm{mmol})$ and 1,2-bis(diphenylphosphino)ethane (40.1 mg, $0.101 \mathrm{mmol})$ in THF $(100 \mathrm{~mL})$ was added a solution of $6(400 \mathrm{mg}, 0.489 \mathrm{mmol})$ in THF $(80 \mathrm{~mL})$ over $1 \mathrm{~h}$. The mixture was stirred for $21 \mathrm{~h}$, and then concentrated in vacuo. The residue was purified by column chromatography on silica gel (EtOAc/hexane, 1:50 to 1:15) to provide $293 \mathrm{mg}$ (84\%) of $\mathbf{1 8}$ as a colorless oil; TLC, $\mathrm{R}_{f} 0.66$ (EtOAc/hexane, 1:4); $[\alpha]^{24}{ }_{\mathrm{D}}+28.8\left(c\right.$ 1.48, $\left.\mathrm{CHCl}_{3}\right) ;{ }^{1} \mathrm{H}-\mathrm{NMR}(270 \mathrm{MHz}) \delta-0.03,0.03,0.05$, 0.06, $0.07(5 \mathrm{~s}$, total $12 \mathrm{H}), 0.86,0.87,0.89,0.90(4 \mathrm{~s}$, total $18 \mathrm{H}), 0.95,0.98(2 \mathrm{~d}, J=6.7 \mathrm{~Hz}$, total $3 \mathrm{H})$, 1.43-1.76 (m, 3H), $1.89(\mathrm{~m}, 1 \mathrm{H}), 2.14(\mathrm{~m}, 1 \mathrm{H}), 2.31-2.46(\mathrm{~m}, 3 \mathrm{H}), 2.46-2.80(\mathrm{~m}, 3 \mathrm{H}), 3.49(\mathrm{~m}, 1 \mathrm{H}) 3.70$, $3.73(2 \mathrm{~s}$, total $3 \mathrm{H}), 3.81(\mathrm{~s}, 3 \mathrm{H}), 3.87(\mathrm{~m}, 1 \mathrm{H}), 4.03(\mathrm{dd}, J=5.2,8.9 \mathrm{~Hz}, 1 \mathrm{H} \times 3 / 5), 4.18,4.22(2 \mathrm{~d}, J=11.6 \mathrm{~Hz}$, total $1 \mathrm{H}), 4.20-4.29(\mathrm{~m}, 1 \mathrm{H}+1 \mathrm{H} \times 2 / 5), 4.49,4.50(2 \mathrm{~d}, J=11.6 \mathrm{~Hz}$, total $1 \mathrm{H}), 5.22(\mathrm{~m}, 1 \mathrm{H}), 5.34-5.48(\mathrm{~m}, 2 \mathrm{H})$, $5.76(\mathrm{~m}, 1 \mathrm{H}), 6.11-6.26(\mathrm{~m}, 1 \mathrm{H}+1 \mathrm{H} \times 3 / 5), 6.48(\mathrm{dd}, J=11.3,15.0 \mathrm{~Hz}, 1 \mathrm{H} \times 2 / 5), 6.88(\mathrm{~m}, 2 \mathrm{H}), 7.25(\mathrm{~m}, 2 \mathrm{H})$; ${ }^{13} \mathrm{C}-\mathrm{NMR}(68 \mathrm{MHz}) \delta-4.67,-4.61,-4.52,-4.35,14.80,15.92,17.82,17.91,18.00,18.14,25.74,25.80$, $25.89,30.72$, 30.78, 36.34, 36.86, 38.56, 39.65, 39.74, 42.04, 44.32, 46.97, 50.39, 50.85, 52.35, 52.41, 55.20, 55.26, 57.65, 58.37, 65.05, 65.86, 68.88, 69.08, 69.48, 69.80, 74.01, 75.30, 113.63, 126.65, 127.57, 128.00, $128.09,129.30,129.38,130.79,130.85,131.08,131.20,131.25,131.31,132.03,132.12,132.23,133.24$, 158.96, 169.44, 169.75, 202.35, 202.76; IR (neat) 2930, 1750, 1650, 1615, 1585, $1515 \mathrm{~cm}^{-1}$; HRMS calcd for $\mathrm{C}_{40} \mathrm{H}_{66} \mathrm{O}_{7} \mathrm{Si}_{2}\left(\mathrm{M}^{+}\right) \mathrm{m} / \mathrm{z} 714.4347$, found 714.4342 . 


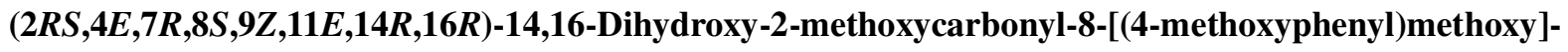
$(2 R S, 4 E, 7 R, 8 S, 9 Z, 11 E, 14 R, 16 R)$-14,16-Dihydroxy-2-methoxycarbonyl-8-[(4-methoxy-phenyl)methoxy]-

7-methyl-4,9,11-cycloheptadecatrien-1-one

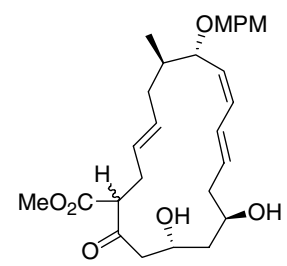

To a cooled $\left(0{ }^{\circ} \mathrm{C}\right)$, stirred solution of $\mathbf{1 8}(73.6 \mathrm{mg}, 0.103 \mathrm{mmol})$ in pyridine $(5 \mathrm{~mL})$ was added HF-pyridine complex $(0.5 \mathrm{~mL})$. The mixture was stirred for $30 \mathrm{~h}$, and quenched with saturated aqueous $\mathrm{NaHCO}_{3}$. This was diluted with saturated aqueous $\mathrm{NaHCO}_{3}(60 \mathrm{~mL})$, and extracted with $\mathrm{CH}_{2} \mathrm{Cl}_{2}$. The combined organic layers were dried and concentrated in vacuo to give the crude diol, which was used in the next step without purification. In a small-scale experiment, a pure sample was obtained by column chromatography on silica gel (EtOAc/hexane, 2:3 to 1:1) as an amorphous solid; TLC, $\mathrm{R}_{f} 0.37$ (acetone/toluene, 1:2), HRMS calcd for $\mathrm{C}_{28} \mathrm{H}_{38} \mathrm{O}_{7}\left(\mathrm{M}^{+}\right) \mathrm{m} / \mathrm{z} 486.2618$, found 486.2618 .

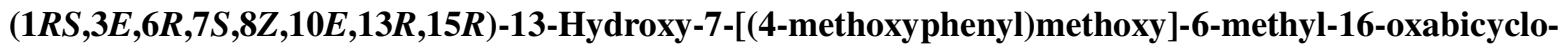
[13.2.2]nonadeca-3,8,10-triene-17,18-dione

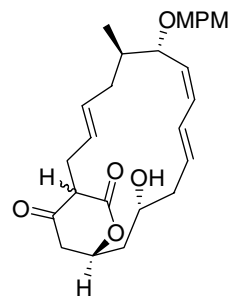

To a cooled $\left(0{ }^{\circ} \mathrm{C}\right)$, stirred solution of the crude diol obtained above in $\mathrm{MeOH}(5 \mathrm{~mL})$ was added $i \mathrm{Pr}_{2} \mathrm{NEt}(0.5$ $\mathrm{mL}$ ). The mixture was stirred for $24 \mathrm{~h}$, and then azeotroped with toluene to give the crude lactone, which was used in the next step without purification. In a small-scale experiment, a pure sample was obtained by column chromatography on silica gel (acetone/toluene, $1: 3$ to $1: 1$ ) as an amorphous solid; TLC, $\mathrm{R}_{f} 0.27$ (acetone/toluene, 1:1), HRMS calcd for $\mathrm{C}_{27} \mathrm{H}_{34} \mathrm{O}_{6}\left(\mathrm{M}^{+}\right) \mathrm{m} / \mathrm{z}$ 454.2355, found 454.2353.

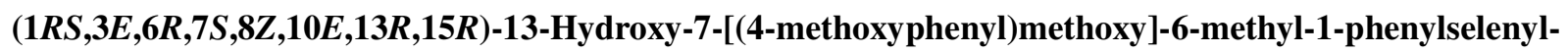
16-oxabicyclo[13.2.2]nonadeca-3,8,10-triene-17,18-dione

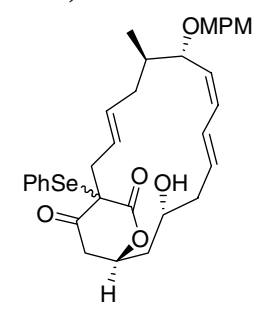

The following reaction was carried out under argon. To a stirred solution of the crude lactone obtained above in $\mathrm{CH}_{2} \mathrm{Cl}_{2}(2 \mathrm{~mL})$ was added $\mathrm{Et}_{3} \mathrm{~N}(0.057 \mathrm{~mL}, 0.41 \mathrm{mmol})$. The mixture was stirred for $10 \mathrm{~min}$, and a solution 
of $\mathrm{PhSeCl}(41.2 \mathrm{mg}, 0.215 \mathrm{mmol})$ in $\mathrm{CH}_{2} \mathrm{Cl}_{2}(0.2 \mathrm{~mL})$ was added at $-78{ }^{\circ} \mathrm{C}$. The mixture was stirred for 30 min at $-78{ }^{\circ} \mathrm{C}$, and then a solution of $\mathrm{PhSeCl}(9.9 \mathrm{mg}, 0.052 \mathrm{mmol})$ in $\mathrm{CH}_{2} \mathrm{Cl}_{2}(0.2 \mathrm{~mL})$ was added. The mixture was stirred for $30 \mathrm{~min}$, and then diluted with toluene $(4 \mathrm{~mL})$ at $-78{ }^{\circ} \mathrm{C}$. The mixture cooled to $-78{ }^{\circ} \mathrm{C}$ was directly transferred into a short column packed with silica gel. The column was eluted with the following cooling (dry ice-acetone bath) solvents (toluene, $\mathrm{CH}_{2} \mathrm{Cl}_{2}$, then EtOAc) successively, to prevent the decomposition of the selenide. The elute was concentrated in vacuo to provide $55.7 \mathrm{mg}$ (89\%, 3 steps from 18) of the phenylselenide as an amorphous solid; TLC, $\mathrm{R}_{f} 0.57$ (acetone/toluene, 1:2), HRMS calcd for $\mathrm{C}_{33} \mathrm{H}_{38} \mathrm{O}_{6} \mathrm{Se}\left(\mathrm{M}^{+}\right) \mathrm{m} / \mathrm{z} 610.1833$, found 610.1842 .

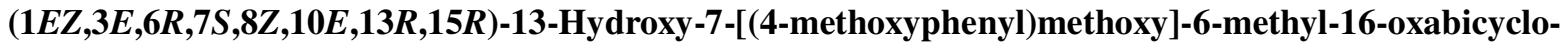

\section{[13.2.2]nonadeca-1,3,8,10-tetraene-17,18-dione (5)}

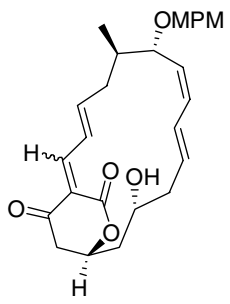

The following reaction was carried out under argon. To a cooled $\left(-50{ }^{\circ} \mathrm{C}\right)$ stirred solution of (1RS,3E,6R,7S,8Z,10E,13R,15R)-13-hydroxy-7-[(4-methoxyphenyl)methoxy]-6-methyl-1-phenylselenyl-16oxa-bicyclo[13.2.2]nonadeca-3,8,10-triene-17,18-dione (159 $\mathrm{mg}, 0.260 \mathrm{~mol}$ ) in $\mathrm{CH}_{2} \mathrm{Cl}_{2}$ (5mL) was added mCPBA (125 mg, $0.724 \mathrm{mmol}$ ). The mixture was stirred for $3.5 \mathrm{~h}$ at $-50{ }^{\circ} \mathrm{C}$, and quenched with $\mathrm{Me}_{2} \mathrm{~S}$. The mixture was stirred for $0.5 \mathrm{~h}$, and then diluted with saturated aqueous $\mathrm{NaHCO}_{3}(20 \mathrm{~mL})$. This was extracted with $\mathrm{CH}_{2} \mathrm{Cl}_{2}$, and the combined organic layers were dried and concentrated in vacuo. The residue was filtered through silica gel to provide $118 \mathrm{mg}$ of $\mathbf{5}$ as a pale yellow oil, which was used in the next step immediately.; TLC, $\mathrm{R}_{f} 0.31$ (acetone/toluene, 1:2), HRMS calcd for $\mathrm{C}_{27} \mathrm{H}_{32} \mathrm{O}_{6}\left(\mathrm{M}^{+}\right) \mathrm{m} / \mathrm{z} 452.2199$, found 452.2195 .

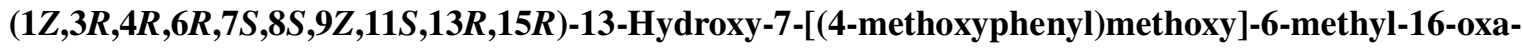
tetracyclo[13.2.2.0 $\left.{ }^{3,11} \cdot 0^{4,8}\right]$ nonadeca-1,9-diene-17,18-dione (4)

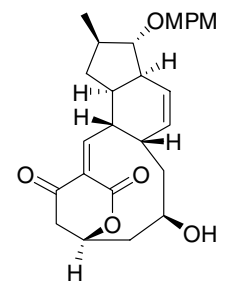

The compound 5 (118 mg, $0.260 \mathrm{mmol})$ was dissolved in degassed toluene $(16 \mathrm{~mL})$, and a crystal of BHT was added. The solution was divided into two $20 \mathrm{~mL}$ sealed tubes equipped with a screwed stopper, and the tubes were filled with argon. The tubes were heated to $130{ }^{\circ} \mathrm{C}$ for $26 \mathrm{~h}$. After being cooled to ambient temperature, the combined solution was concentrated in vacuo. The residue was purified by column chromatography on silica gel (EtOAc/toluene, 1:2) to provide $62.1 \mathrm{mg}$ (53\%, 2 steps from the 
phenylselenide) of 4 as a colorless oil; TLC, $\mathrm{R}_{f} 0.31$ (acetone/toluene, 1:2); $[\alpha]^{23}{ }_{\mathrm{D}}+37.5\left(c 0.625, \mathrm{CHCl}_{3}\right)$; ${ }^{1} \mathrm{H}-\mathrm{NMR}(270 \mathrm{MHz}) \delta 1.08(\mathrm{~d}, J=6.6 \mathrm{~Hz}, 3 \mathrm{H}), 1.48(\mathrm{q}, J=12.1 \mathrm{~Hz}, 1 \mathrm{H}), 1.64-1.87(\mathrm{~m}, 3 \mathrm{H}), 1.90-2.06(\mathrm{~m}$, 2H), 2.42-2.57 (m, 2H), $2.66(\mathrm{dd}, J=0.7,18.7 \mathrm{~Hz}, 1 \mathrm{H}), 2.70-2.83(\mathrm{~m}, 2 \mathrm{H}), 3.12$ (ddd, $J=0.7,7.3,18.7 \mathrm{~Hz}$, $1 \mathrm{H}), 3.26(\mathrm{dd}, J=2.7,6.8 \mathrm{~Hz}, 1 \mathrm{H}), 3.32(\mathrm{~m}, 1 \mathrm{H}), 3.65(\mathrm{~m}, 1 \mathrm{H}), 3.81(\mathrm{~s}, 3 \mathrm{H}), 4.44,4.57(2 \mathrm{~d}, J=11.2 \mathrm{~Hz}$, $1 \mathrm{H} \times 2), 4.89(\mathrm{~m}, 1 \mathrm{H}), 5.68(\mathrm{~d}, J=10.4 \mathrm{~Hz}, 1 \mathrm{H}), 5.73(\mathrm{~d}, J=10.4 \mathrm{~Hz}, 1 \mathrm{H}), 6.86-6.91(\mathrm{~m}, 3 \mathrm{H}), 7.29(\mathrm{~m}, 2 \mathrm{H})$; ${ }^{13} \mathrm{C}-\mathrm{NMR}(68 \mathrm{MHz}) \delta 18.69,35.22,35.53,37.61,39.62,39.88,41.26,41.32,41.75,45.61,55.29,66.86$, 71.53, 72.71, 92.38, 113.83×2, 124.83, 129.27×2, 130.50, 131.02, 135.95, 157.03, 159.16, 165.72, 194.23; IR (neat) 3450, 2950, 1735, 1705, 1620, $1515 \mathrm{~cm}^{-1}$; HRMS calcd for $\mathrm{C}_{27} \mathrm{H}_{32} \mathrm{O}_{6}\left(\mathrm{M}^{+}\right) \mathrm{m} / \mathrm{z} 452.2199$, found 452.2195 .

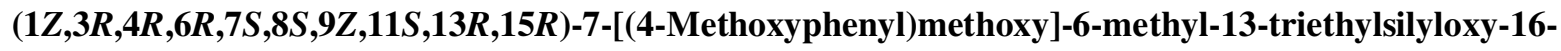
oxatetracyclo[13.2.2.0 $\left.{ }^{3,11} \cdot 0^{4,8}\right]$ nonadeca-1,9-diene-17,18-dione

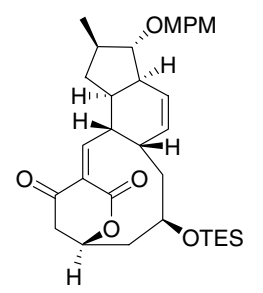

The following reaction was carried out under argon. To a cooled $\left(-78{ }^{\circ} \mathrm{C}\right)$, stirred solution of $4(38.6 \mathrm{mg}$, $0.0853 \mathrm{mmol})$ in $\mathrm{CH}_{2} \mathrm{Cl}_{2}(4 \mathrm{~mL})$ were added 2,6-lutidine $(0.10 \mathrm{~mL}, 0.85 \mathrm{mmol})$ and TESOTf $(0.10 \mathrm{~mL}, 0.43$ mmol). The mixture was stirred at $-78{ }^{\circ} \mathrm{C}$ for $0.5 \mathrm{~h}$, and quenched with saturated aqueous $\mathrm{NaHCO}_{3}$. This was diluted with saturated aqueous $\mathrm{NaHCO}_{3}(10 \mathrm{~mL})$, and extracted with $\mathrm{CH}_{2} \mathrm{Cl}_{2}$. The combined organic layers were dried and concentrated in vacuo. The residue was purified by column chromatography on silica gel (EtOAc/hexane, 1:4) to provide $35.9 \mathrm{mg}$ (78\%) of the TES ether as a colorless oil; TLC, $\mathrm{R}_{f} 0.33$ (EtOAc/hexane, 1:2); $[\alpha]^{23}{ }_{\mathrm{D}}+47.1\left(c 0.40, \mathrm{CHCl}_{3}\right) ;{ }^{1} \mathrm{H}-\mathrm{NMR}(270 \mathrm{MHz}) \delta$ 0.49-0.56 (m, 6H), 0.90-0.10 (m, 9H), $1.07(\mathrm{~d}, J=6.6 \mathrm{~Hz}, 3 \mathrm{H}), 1.25(\mathrm{q}, J=11.7 \mathrm{~Hz}, 1 \mathrm{H}), 1.62(\mathrm{~m}, 1 \mathrm{H}), 1.72(\mathrm{~m}, 1 \mathrm{H}), 1.82(\mathrm{~m}, 1 \mathrm{H}), 1.95-2.12$ $(\mathrm{m}, 2 \mathrm{H}), 2.39-2.54(\mathrm{~m}, 2 \mathrm{H}), 2.62(\mathrm{dd}, J=0.7,18.5 \mathrm{~Hz}, 1 \mathrm{H}), 2.77-2.89(\mathrm{~m}, 2 \mathrm{H}), 3.12(\mathrm{dd}, J=7.2,18.5 \mathrm{~Hz}$, $1 \mathrm{H}), 3.27(\mathrm{dd}, J=2.6,6.6 \mathrm{~Hz}, 1 \mathrm{H}), 3.31(\mathrm{~m}, 1 \mathrm{H}), 3.68(\mathrm{~m}, 1 \mathrm{H}), 3.81(\mathrm{~s}, 3 \mathrm{H}), 4.45,4.57(2 \mathrm{~d}, J=11.2 \mathrm{~Hz}$, $1 \mathrm{H} \times 2), 4.87(\mathrm{~m}, 1 \mathrm{H}), 5.65(\mathrm{~d}, J=10.4 \mathrm{~Hz}, 1 \mathrm{H}), 5.71(\mathrm{~d}, J=10.4 \mathrm{~Hz}, 1 \mathrm{H}), 6.90(\mathrm{~d}, J=11.4 \mathrm{~Hz}, 1 \mathrm{H}), 6.90(\mathrm{~m}$, $2 \mathrm{H}), 7.30(\mathrm{~m}, 2 \mathrm{H}) ;{ }^{13} \mathrm{C}-\mathrm{NMR}(68 \mathrm{MHz}) \delta 5.35 \times 3,6.88 \times 3,18.86,35.56 \times 2,37.64,39.65,39.82,41.47,41.81$, 42.30, 45.61, 55.26, 67.79, 71.59, 72.77, 92.61, 113.80×2, 125.52, 129.27×2, 130.27, 130.59, 135.86, 157.23, 159.16, 165.75, 194.03; IR (neat) 2950, 1740, 1715, 1615, $1515 \mathrm{~cm}^{-1}$; HRMS calcd for $\mathrm{C}_{33} \mathrm{H}_{46} \mathrm{O}_{6} \mathrm{Si}\left(\mathrm{M}^{+}\right) \mathrm{m} / \mathrm{z}$ 566.3064 , found 566.3061 . 


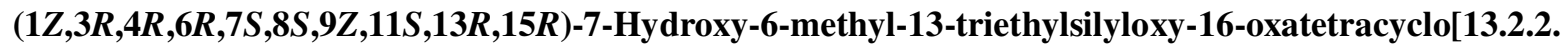
$\left.0^{3,11} .0^{4,8}\right]$ nonadeca-1,9-diene-17,18-dione

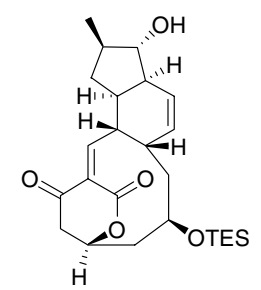

To a stirred solution of $(1 Z, 3 R, 4 R, 6 R, 7 S, 8 S, 9 Z, 11 S, 13 R, 15 R)-7$-[(4-methoxyphenyl)methoxy]-6-methyl-13triethylsilyloxy-16-oxatetracyclo[13.2.2.0 $\left.0^{3,11} .0^{4,8}\right]$ nonadeca-1,9-diene-17,18-dione (35.9 mg, $\left.0.0633 \mathrm{mmol}\right)$ in $\mathrm{CH}_{2} \mathrm{Cl}_{2}(4 \mathrm{~mL})$ was added aqueous phosphate buffer $(0.4 \mathrm{~mL}, \mathrm{pH}$ 7). This was stirred for $30 \mathrm{~min}$ and then DDQ $(22.4 \mathrm{mg}, 0.0985 \mathrm{mmol})$ was added at $0{ }^{\circ} \mathrm{C}$. The mixture was stirred for $3.5 \mathrm{~h}$ and then DDQ $(18.9 \mathrm{mg}$, $0.0831 \mathrm{mmol}$ ) was added at $0{ }^{\circ} \mathrm{C}$. The mixture was stirred for $2 \mathrm{~h}$ and then quenched with saturated aqueous $\mathrm{NaHCO}_{3}$. This was diluted with saturated aqueous $\mathrm{NaHCO}_{3}(15 \mathrm{~mL})$, and extracted with $\mathrm{CH}_{2} \mathrm{Cl}_{2}$. The combined organic layers were dried and concentrated in vacuo. The residue was purified by column chromatography on silica gel (EtOAc/hexane, 1:3 to 1:1) to provide $26.1 \mathrm{mg}(92 \%)$ of the secondary alcohol as a colorless oil; TLC, $\mathrm{R}_{f} 0.30$ (EtOAc/hexane, 1:1); $[\alpha]^{22}{ }_{\mathrm{D}}+65.9\left(c 0.33, \mathrm{CHCl}_{3}\right) ;{ }^{1} \mathrm{H}-\mathrm{NMR}(270 \mathrm{MHz})$ $\delta$ 0.49-0.58 (m, 6H), 0.89-0.95 (m, 9H), $1.07(\mathrm{~d}, J=6.6 \mathrm{~Hz}, 3 \mathrm{H}), 1.25(\mathrm{~m}, 1 \mathrm{H}), 1.61-1.87(\mathrm{~m}, 3 \mathrm{H}), 2.08(\mathrm{~m}$, $1 \mathrm{H}), 2.40-2.66(\mathrm{~m}, 4 \mathrm{H}), 2.62(\mathrm{dd}, J=0.7,18.7 \mathrm{~Hz}, 1 \mathrm{H}), 2.80(\mathrm{~m}, 1 \mathrm{H}), 3.12(\mathrm{ddd}, J=0.7,7.3,18.7 \mathrm{~Hz}, 1 \mathrm{H})$, $3.28(\mathrm{ddd}, J=1.5,5.9,11.4 \mathrm{~Hz}, 1 \mathrm{H}), 3.44(\mathrm{dd}, J=4.0,6.6 \mathrm{~Hz}, 1 \mathrm{H}), 3.69(\mathrm{~m}, 1 \mathrm{H}), 4.87(\mathrm{~m}, 1 \mathrm{H}), 5.74(\mathrm{~d}, J=$ $10.4 \mathrm{~Hz}, 1 \mathrm{H}), 5.85(\mathrm{dt}, J=10.4,2.9 \mathrm{~Hz}, 1 \mathrm{H}), 6.86(\mathrm{~d}, J=11.4 \mathrm{~Hz}, 1 \mathrm{H}) ;{ }^{13} \mathrm{C}-\mathrm{NMR}(68 \mathrm{MHz}) \delta 5.35 \times 3$, $6.88 \times 3,17.94,35.53,35.73,36.69,40.20,41.41,41.81,42.33,44.29,45.64,67.81,72.80,86.47,125.18$, 125.44, 129.90, 157.11, 165.75, 193.97; IR (neat) 3440, 2950, 1730, 1715, $1615 \mathrm{~cm}^{-1}$; HRMS calcd for $\mathrm{C}_{25} \mathrm{H}_{38} \mathrm{O}_{5} \mathrm{Si}\left(\mathrm{M}^{+}\right) \mathrm{m} / \mathrm{z} 446.2489$, found 446.2497.

$(1 Z, 3 R, 4 R, 6 R, 8 S, 9 Z, 11 S, 13 R, 15 R)-6$-methyl-13-triethylsilyloxy-16-oxatetracyclo[13.2.2.0 $\left.0^{3,11} .0^{4,8}\right]$ nonadeca-1,9-diene-7,17,18-trione

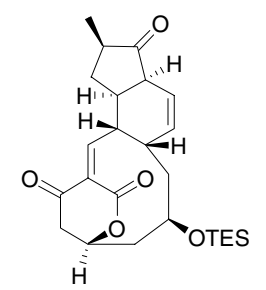

To a cooled $\left(0{ }^{\circ} \mathrm{C}\right)$, stirred solution of $(1 Z, 3 R, 4 R, 6 R, 7 S, 8 S, 9 Z, 11 S, 13 R, 15 R)$-7-hydroxy-6-methyl-13-triethylsilyloxy-16-oxatetracyclo[13.2.2.0 $\left.0^{3,11} \cdot 0^{4,8}\right]$ nonadeca-1,9-diene-17,18-dione (23.5 mg, $\left.0.0526 \mathrm{mmol}\right)$ in $\mathrm{CH}_{2} \mathrm{Cl}_{2}(3 \mathrm{~mL})$ were added $\mathrm{NaHCO}_{3}(14.4 \mathrm{mg}, 0.171 \mathrm{mmol})$ and Dess-Martin periodinane $(34.4 \mathrm{mg}, 0.0810$ mmol). The mixture was stirred for $1.5 \mathrm{~h}$ and then diluted with saturated aqueous $\mathrm{NaHCO}_{3}-20 \%$ aqueous $\mathrm{Na}_{2} \mathrm{~S}_{2} \mathrm{O}_{3}(1: 1,10 \mathrm{~mL})$ and $\mathrm{CH}_{2} \mathrm{Cl}_{2}(5 \mathrm{~mL})$ at $0{ }^{\circ} \mathrm{C}$. The mixture was stirred for $30 \mathrm{~min}$ and then the organic layer was separated. The aqueous layer was extracted with $\mathrm{CH}_{2} \mathrm{Cl}_{2}$. The combined organic layers were dried 
and concentrated in vacuo. The residue was purified by column chromatography on silica gel (EtOAc/hexane, 1:3) to provide $22.0 \mathrm{mg}(94 \%)$ of the ketone as a colorless oil; TLC, $\mathrm{R}_{f} 0.55$ (EtOAc/hexane, 1:1); $[\alpha]^{23}$ +182 (c 0.26, $\left.\mathrm{CHCl}_{3}\right) ;{ }^{1} \mathrm{H}-\mathrm{NMR}(270 \mathrm{MHz}) \delta$ 0.47-0.56 (m, 6H), 0.88-0.94 (m, 9H), $1.11(\mathrm{~d}, J=6.6 \mathrm{~Hz}, 3 \mathrm{H})$, $1.47(\mathrm{q}, J=12.1 \mathrm{~Hz} 1 \mathrm{H}), 1.68-1.74(\mathrm{~m}, 2 \mathrm{H}), 2.12(\mathrm{dt}, J=16.5,6.6 \mathrm{~Hz}, 1 \mathrm{H}), 2.21-2.35(\mathrm{~m}, 2 \mathrm{H}), 2.46(\mathrm{ddd}, J$ $=4.2,11.5,15.6 \mathrm{~Hz}, 1 \mathrm{H}), 2.63(\mathrm{~m}, 1 \mathrm{H}), 2.64(\mathrm{dd}, J=0.7,18.7 \mathrm{~Hz}, 1 \mathrm{H}), 2.88(\mathrm{~m}, 1 \mathrm{H}), 3.14(\mathrm{ddd}, J=0.7,7.3$, $18.7 \mathrm{~Hz}, 1 \mathrm{H}), 3.15(\mathrm{~m}, 1 \mathrm{H}), 3.44(\mathrm{ddd}, J=2.2,6.2,11.7 \mathrm{~Hz}, 1 \mathrm{H}), 3.67(\mathrm{~m}, 1 \mathrm{H}), 4.89(\mathrm{~m}, 1 \mathrm{H}), 5.66(\mathrm{dt}, J=$ $10.3,3.1 \mathrm{~Hz}, 1 \mathrm{H}), 5.88(\mathrm{dt}, J=10.3,2.2 \mathrm{~Hz}, 1 \mathrm{H}), 6.81(\mathrm{~d}, J=11.7 \mathrm{~Hz}, 1 \mathrm{H}) ;{ }^{13} \mathrm{C}-\mathrm{NMR}(68 \mathrm{MHz}) \delta 5.33 \times 3$, $6.85 \times 3,13.96,33.17,34.67,35.53,38.56,41.47,41.98,43.88,45.53,45.67,67.70,72.85,123.02$, 128.32, 136.32, 155.36, 165.67, 193.91, 217.13; IR (neat) 2960, 1740, 1700, $1620 \mathrm{~cm}^{-1}$; HRMS calcd for $\mathrm{C}_{25} \mathrm{H}_{36} \mathrm{O}_{5} \mathrm{Si}$ $\left(\mathbf{M}^{+}\right) \mathrm{m} / \mathrm{z}$ 444.2332, found 444.2331 .

\section{(+)-Macquarimicin A (1)}

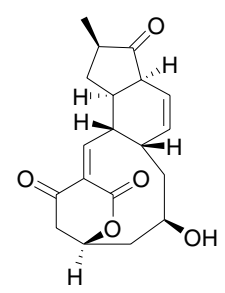

To a cooled $\left(0{ }^{\circ} \mathrm{C}\right)$, stirred solution of $(1 Z, 3 R, 4 R, 6 R, 8 S, 9 Z, 11 S, 13 R, 15 R)$-6-methyl-13-triethylsilyloxy-16oxatetracyclo[13.2.2. $\left.0^{3,11} \cdot 0^{4,8}\right]$ nonadeca-1,9-diene-7,17,18-trione (22.0 mg, $\left.0.0495 \mathrm{mmol}\right)$ in $\mathrm{MeOH}(3 \mathrm{~mL})$ was added PPTS (1.0 mg, $0.0040 \mathrm{mmol})$. The mixture was stirred for $2 \mathrm{~h}$ and then $\mathrm{NaHCO}_{3}(6.0 \mathrm{mg}, 0.071$ mmol) was added at $0{ }^{\circ} \mathrm{C}$. This was azeotroped with EtOAc and the residue was purified by column chromatography on silica gel (EtOAc/toluene, 1:1) to provide $15.8 \mathrm{mg}(97 \%)$ of macquarimicin $\mathrm{A}(\mathbf{1})$ as an amorphous solid; TLC, $\mathrm{R}_{f} 0.45$ (acetone/toluene, 2:3); $[\alpha]_{\mathrm{D}}^{23}+270(c 0.20, \mathrm{MeOH}),[\alpha]^{23}{ }_{\mathrm{D}}+285(c 0.780$, $\mathrm{MeOH}) ;{ }^{1} \mathrm{H}-\mathrm{NMR}(270 \mathrm{MHz}) \delta 1.11(\mathrm{~d}, J=6.6 \mathrm{~Hz}, 3 \mathrm{H}), 1.48(\mathrm{q}, J=12.1 \mathrm{~Hz}, 1 \mathrm{H}), 1.75-1.81(\mathrm{~m}, 2 \mathrm{H}), 2.01$ $(\mathrm{dt}, J=16.9,6.6 \mathrm{~Hz}, 1 \mathrm{H}), 2.19-2.36(\mathrm{~m}, 2 \mathrm{H}), 2.51(\mathrm{ddd}, J=4.0,11.7,15.4 \mathrm{~Hz}, 1 \mathrm{H}), 2.64(\mathrm{~m}, 1 \mathrm{H}), 2.68(\mathrm{dd}, J$ $=0.7,19.1 \mathrm{~Hz}, 1 \mathrm{H}), 2.90(\mathrm{~m}, 1 \mathrm{H}), 3.15(\mathrm{ddd}, J=0.9,7.5,19.1 \mathrm{~Hz}, 1 \mathrm{H}), 3.15(\mathrm{~m}, 1 \mathrm{H}), 3.46(\mathrm{ddd}, J=2.3,6.0$, $11.4 \mathrm{~Hz}, 1 \mathrm{H}), 3.65(\mathrm{~m}, 1 \mathrm{H}), 4.92(\mathrm{~m}, 1 \mathrm{H}), 5.67(\mathrm{dt}, J=10.3,2.9 \mathrm{~Hz}, 1 \mathrm{H}), 5.91(\mathrm{dt}, J=10.3,2.0 \mathrm{~Hz}, 1 \mathrm{H}), 6.80$ $(\mathrm{d}, J=11.4 \mathrm{~Hz}, 1 \mathrm{H}) ;{ }^{13} \mathrm{C}-\mathrm{NMR}(68 \mathrm{MHz}) \delta 13.91,33.17,34.67,35.25,38.64,40.86,41.26,43.88,45.64$, 45.70, 66.81, 72.77, 123.68, 127.57, 136.44, 155.15, 165.64, 194.12, 217.04; IR (neat) 3450, 2930, 1735, $1705,1620 \mathrm{~cm}^{-1}$; HRMS calcd for $\mathrm{C}_{19} \mathrm{H}_{22} \mathrm{O}_{5}\left(\mathrm{M}^{+}\right) \mathrm{m} / \mathrm{z} 330.1467$, found 330.1457 . 


\section{Determination of the geometry of the $\mathrm{C}(2)-\mathrm{C}(3)$ double bond}

The relative configuration of macquarimicin A (1) was originally assigned based on that of macquarimicin $\mathrm{B}$, which was determined by a single crystal X-ray experiment (Fig. S1). ${ }^{3}$ Since the $\mathrm{C}(2)-\mathrm{C}(3)$ double bond does not exist in macquarimicin $\mathrm{B}$, there are no experimental data that support the reported $E$ geometry of the double bond. Considering the structural and spectroscopic similarity of $\mathbf{1}$ to cochleamycin A (2), whose $\mathrm{C}(4)-\mathrm{C}(5)$ geometry (cochleamycin numbering) was determined by a ${ }^{13} \mathrm{C}-\left\{{ }^{1} \mathrm{H}\right\}$ NOE experiment, ${ }^{4}$ it is expected that the $\mathrm{C}(2)-\mathrm{C}(3)$ geometry of $\mathbf{1}$ may be $Z$. To establish the stereochemistry and $\mathrm{C}(2)-\mathrm{C}(3)$ geometry of 1 umambiguously, we conducted the throughout NOE experiments on our synthetic sample, which lead to the confirmation of the relative configuration of $\mathbf{1}$ and the revision of $\mathrm{C}(2)-\mathrm{C}(3)$ geometry to be $Z$ (Fig. S2). The observed NOEs and $J$ values are completely in accordance with the conformation depicted in Fig. S2. None of other diastereomeric or geometrical isomers, including 1' (Fig. S3), can explain these results.
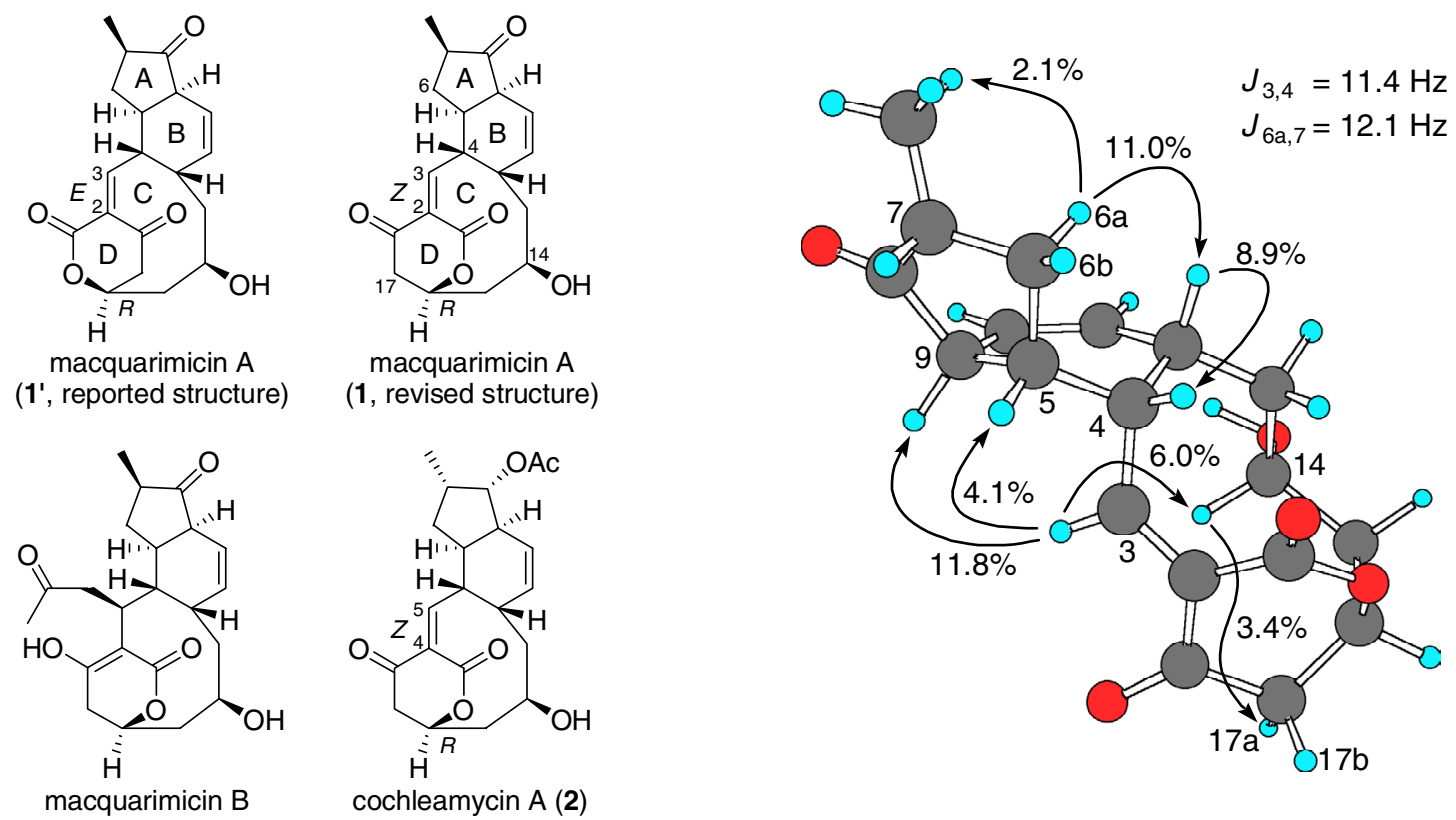

Fig. S1. Structures of macquarimicins A and $\mathrm{B}$, and cochleamycin $\mathrm{A}$.

Fig. S2. NOE experiments on macquarimicin A.

${ }^{3}$ Hochlowski, J. E.; Mullally, M. M.; Henry, R.; Whittern, D. M.; McAlpine, J. B. J. Antibiot. 1995, 48, 467.

${ }^{4}$ Shindo, K.; Iijima, H.; Kawai, H. J. Antibiot. 1996, 49, 244. 

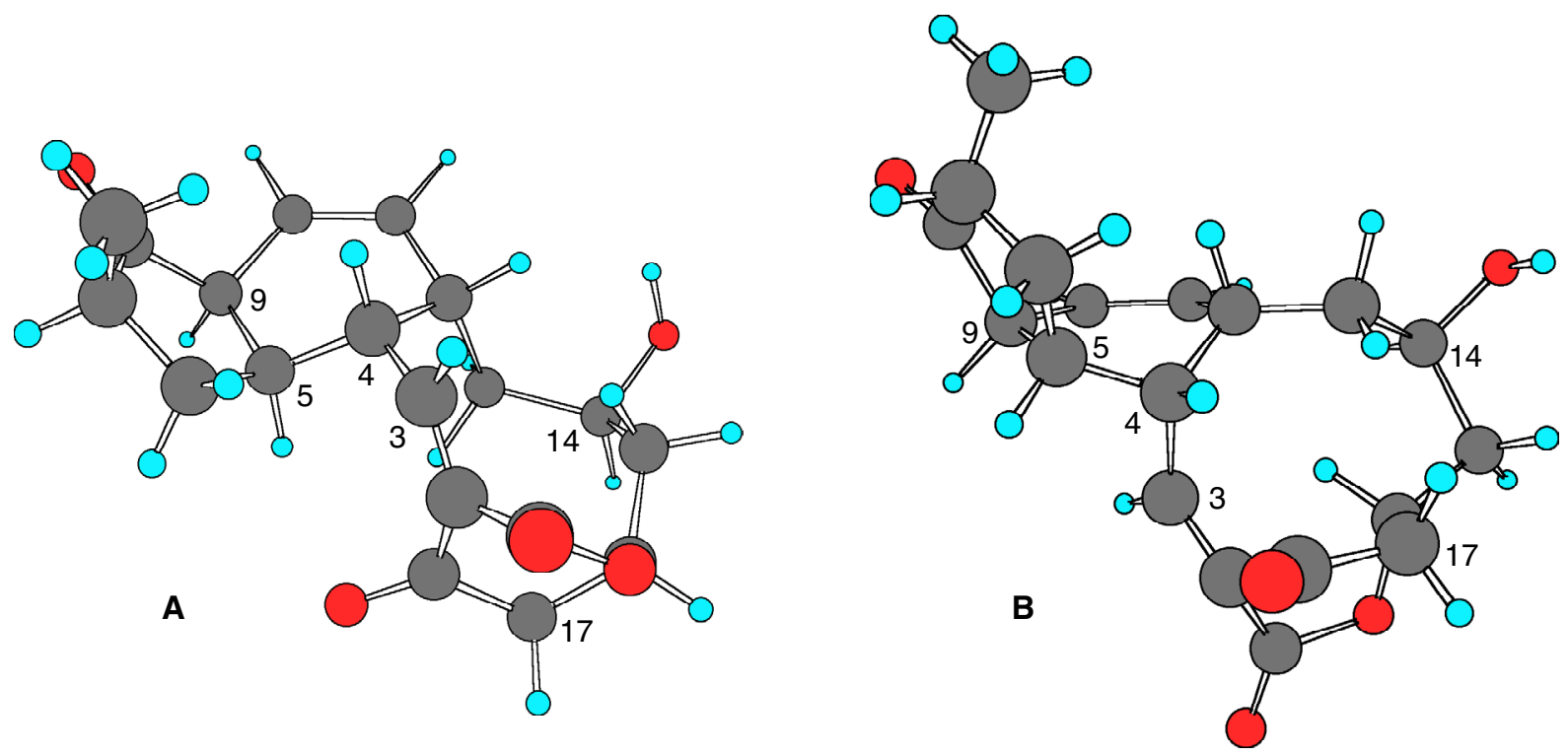

Fig. S3. Two possible conformers of $(E)$-macquarimicin A (1'). 